\title{
The Area of Horizons and the Trapped Region
}

\author{
Lars Andersson $^{1,2, \star}$, Jan Metzger ${ }^{1,3, \star \star}$ \\ 1 Albert-Einstein-Institut, Am Mühlenberg 1, 14476 Potsdam, Germany. \\ E-mail: lars.andersson@aei.mpg.de; jan.metzger@aei.mpg.de \\ 2 Department of Mathematics, University of Miami, Coral Gables, FL 33124, USA \\ 3 Stanford University, Mathematics, 450 Serra Mall, Stanford, CA 94305, USA
}

Received: 23 September 2008 / Accepted: 16 October 2008

Published online: 24 January 2009 - () The Author(s) 2009. This article is published with open access at Springerlink.com

\begin{abstract}
This paper considers some fundamental questions concerning marginally trapped surfaces, or apparent horizons, in Cauchy data sets for the Einstein equation. An area estimate for outermost marginally trapped surfaces is proved. The proof makes use of an existence result for marginal surfaces, in the presence of barriers, curvature estimates, together with a novel surgery construction for marginal surfaces. These results are applied to characterize the boundary of the trapped region.
\end{abstract}

\section{Introduction}

Trapped and marginally trapped surfaces play a central role in the analysis of spacetime geometry. By the singularity theorems of Hawking and Penrose [HE73], a spacetime which satisfies suitable energy and causality conditions, and which in addition contains a trapped surface, must contain a black hole. Marginally trapped surfaces, or apparent horizons, serve as the quasi-local version of black hole boundary. In numerical general relativity, they are used as excision surfaces for the evolution of black hole initial data, and approximations to physical characteristics of a black hole such as linear and angular momentum [KLZ07, CLZ ${ }^{+}$07] can be calculated in terms of data induced on the apparent horizon.

We briefly recall some basic facts. A two dimensional spacelike surface $\Sigma$ in a 4-dimensional Lorentzian spacetime has, up to normalization, two future pointing null normals. We designate one of these, $\ell^{+}$, the outward pointing, and the other $\ell^{-}$, the inward pointing null normal. Corresponding to $\ell^{ \pm}$we have the null mean curvatures or null expansions $\theta^{ \pm}$. Let $(M, g, K)$ be a Cauchy data set containing $\Sigma$. Then $\theta^{ \pm}$is given by

$$
\theta^{ \pm}=P \pm H,
$$

* Supported in part by the NSF, under contract no. DMS 0407732 with the University of Miami.

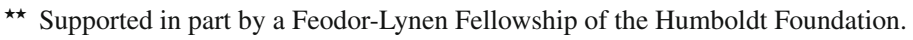


where $H$ is the mean curvature of $\Sigma$ in $M$ with respect to the outward pointing normal, and $P=\operatorname{tr}^{\Sigma} K$, the trace of the projection of $K$ to $\Sigma$. The surface $\Sigma$ is said to be (future) trapped if $\theta^{ \pm}<0$, and (future) marginally trapped if $\theta^{-}<0$, while $\theta^{+}=0$. If $\theta^{+}<0$ or $\theta^{+}>0$, with no condition imposed on $\theta^{-}$, then $\Sigma$ is called outer trapped or outer untrapped, respectively. Finally, if the condition $\theta^{+}=0$ holds, with no further condition on $\theta^{-}$, then $\Sigma$ is called a marginally outer trapped surface, or MOTS. We will explicitly review notation and further conditions needed on $(M, g, K)$ in Sect. 2.

From a mathematical point of view, MOTS are the natural generalization of minimal surfaces to a Lorentzian setting, see the discussion in [AM05]. In particular, in the case of time-symmetric Cauchy data, where $K \equiv 0$, a MOTS is a minimal surface. However, a fundamental difference between minimal surfaces and MOTS, is that MOTS are not stationary with respect to an elliptic functional. In spite of this, there is a notion of stability for MOTS analogous to the notion of stability for minimal surfaces, cf. [AMS05,AMS07]. Although the stability operator in the case of MOTS fails to be self-adjoint, many of the results and ideas generalize from the case of stable minimal surfaces to the case of stable MOTS. In particular, a curvature estimate, generalizing the classical result of [SSY75] was proved in [AM05] for the case of stable MOTS.

The so-called Jang's equation [Jan78] is closely related to the equation $\theta^{+}=0$. Both are prescribed mean curvature equations, where the right hand side depend on the normal. A careful study of Jang's equation is a crucial ingredient in the positive mass proof of Schoen and Yau [SY81]. Among other things, their argument makes use of the fact that the boundary of the blowup set for Jang's equation consists of marginal surfaces. This means that the question of existence of MOTS may be approached by studying the existence of blowup solutions to Jang's equation. This observation was used by Yau [Yau01] to give a criterion for a Cauchy data set to contain a marginal surface.

A consequence of the fact that MOTS are not critical points for a variational principle is that the familiar barrier arguments for the existence of minimal surfaces do not generalize to MOTS. However, as was pointed out by Schoen in a talk given at the Miami Waves conference in 2004 [Sch04], the fact that blowup surfaces for Jang's equation are marginal surfaces actually provides a result which replaces the above mentioned barrier arguments.

Theorem 1.1. Let $(M, g, K)$ be a Cauchy data set. Assume that $M$ is compact with two boundary components, an inner and an outer boundary and assume that the inner boundary is outer trapped and the outer boundary is outer untrapped. Then $M$ contains a stable MOTS.

This theorem follows from Schoen's original result, stated as Theorem 3.1 and a closer analysis of the blow-up surface, cf. Theorem 4.1. Unfortunately, a proof of Theorem 3.1 has not been published. In Sect. 3 we therefore prove this result in detail, since it is crucial for the results in this paper.

We wish to remark here that if the ambient manifold is asymptotically flat with appropriate fall-off conditions, then spheres near infinity will be untrapped and can serve as outer barriers in Theorem 1.1.

Starting from the curvature estimates for MOTS mentioned above, it is easy to show that the set of all stable marginally trapped surfaces in a compact region is compact, given a uniform estimate for the area. However, such an estimate cannot be expected to hold in general. Examples due to Colding-Minicozzi and others [CM99,Dea03] show that for each genus $g \geq 1$ there is an example of a compact three dimensional manifold containing a sequence of stable minimal surfaces of genus $g$ with unbounded area. 
Recalling that minimal surfaces are MOTS in the special case $K=0$, this shows that an a priori area estimate for MOTS requires further conditions.

If we consider surfaces minimizing area in a given homology class, on the other hand, there is no need to prove an area bound to obtain compactness, as one can assume that the area is bounded by the area of any comparison surface. For the case of MOTS, the appropriate analogue of a minimizing surface is an outermost MOTS. We say that a MOTS $\Sigma$ is outermost in $M$ if there is no other MOTS in the complement of the region which $\Sigma$ bounds with a, possibly empty, inner boundary. In this respect, the main result of this paper, cf. Theorem 6.5 is an area estimate for the outermost MOTS.

Theorem 1.2. There exists a constant $C$ which is an increasing function of $\left\|^{M} \mathrm{Rm}\right\|_{C^{0}(M)}$, $\|K\|_{C^{1}(M)}$, $\operatorname{inj}_{\rho}(M, g, K ; \partial M)^{-1}$, and $\mathrm{Vol} M$ such that the area of an outermost MOTS $\Sigma$ satisfies the estimate

$$
|\Sigma| \leq C
$$

The quantity $\operatorname{inj}_{\rho}(M, g, K ; \partial M)$ is explained in Definition 2.8.

This result does not require the MOTS to be connected. Thus, in combination with the curvature estimate for stable MOTS we infer an estimate for the number of components of the outermost MOTS.

Note, even for outward minimizing surfaces the above bound does not actually follow from the variational principle, as it does not refer to the area of a comparison surface. In this respect our area estimate is related to the area estimate in [NR06] for minimizing minimal surfaces in terms of volume and the homologial filling functions of the ambient manifold, which must have simple enough homology.

To put Theorem 1.2 into perspective, recall that the Penrose inequality is a conjectured relation between the ADM mass and the area of the horizon. For a general Cauchy data set, the exact statement of the Penrose inequality is a subtle issue. Although, the area estimate stated in Theorem 1.2 holds for outermost MOTS, a counterexample due to Ben-Dov [BD04] shows that an inequality between the area of the outermost MOTS and the ADM mass does not hold in general.

One of the main steps in the proof of Theorem 1.2 is a surgery argument, which is given in Sect. 6. This argument constructs, given a stable MOTS $\Sigma$ with sufficiently large area and an outer barrier surface, another stable MOTS outside $\Sigma$. The two main steps in the argument is to show, using the curvature estimate, that given a stable MOTS with sufficiently large area, it is possible to glue in a neck with negative $\theta^{+}$, thereby constructing a $\Sigma^{\prime}$ outside $\Sigma$ with $\theta^{+} \leq 0$. Together with Theorem 1.1 this yields a contradiction to the assumption that $\Sigma$ is outermost.

The surgery argument may also be used to give a replacement for the strong maximum principle for outermost MOTS. It should be noted that for general MOTS, the strong maximum principle does not apply in general, in particular it can not be used to rule out that a surface touches itself in points where the normals of the two touching pieces point into opposite directions. This is exactly the situation which we can address with the surgery argument.

Combining the above area estimate for outermost MOTS and the curvature estimate of [AM05] yields, as already mentioned, a compactness result for the class of outermost MOTS in a compact region. Using this fact in combination with the surgery technique discussed above enables us to give a characterization of the boundary of the trapped region. 
The outer trapped region is the union of all domains bounded by a weakly outer trapped surface and the, possibly empty, interior boundary of the initial data set. It has been proposed by several authors that the boundary of the outer trapped region is a smooth MOTS. However, the arguments put forth to prove this, see for example [HE73, KH97], relied on strong extra assumptions such as a piecewise smoothness of the boundary. Using the techniques developed in this paper we are able to settle this problem completely.

Theorem 1.3. The boundary of the outer trapped region is a smooth outermost MOTS. Furthermore, it is the unique outermost MOTS.

The boundary of the outer trapped region is defined and examined in Sect. 7, where Theorem 7.3 is proved, a more precise version of Theorem 1.3. The main idea here is that barrier constructions using a smoothing result from Kriele-Hayward [KH97], cf. Lemma 2.14, and Theorem 5.1 can be used to prove a replacement for the maximum principle for outermost MOTS. Together with the compactness properties for stable MOTS, and the area estimate for outermost MOTS, this gives the result.

Although the presentation here is restricted to the $n=3$ dimensional case, most of the techniques proposed generalize to higher dimensions. The points which need to be addressed in the higher dimensional case are regularity issues for Jang's equation, cf. Remark 3.2, and the a priori curvature estimates for stable MOTS used in the surgery procedure of Sect. 6. See [Eic07] for a treatment of these issues in the higher dimensional case.

\section{Preliminaries}

An initial data set for the Einstein equations is a 3-dimensional Riemannian manifold $(M, g)$ together with a symmetric two-tensor $K$ representing the second fundamental form of $M$ viewed as a Cauchy hypersurface in a four dimensional spacetime. In this paper we will not make further use of the spacetime geometry and in particular, energy conditions or constraint equations on $(g, K)$ are not needed for this paper.

A surface in $M$ is called two-sided if its normal bundle is orientable, i.e. if it is possible to choose a globally defined normal. As there are two such choices we will assume that there is one distinguished direction which we call the outer normal. We will denote this outer normal vector field by $\nu$.

Given a two-sided surface $\Sigma$ in $M$, we denote its second fundamental form, defined with respect to its outer normal $v$, by $A$. Further, we denote by $H, P$ the mean curvature, $H={ }^{\Sigma}$ div $v$, and the trace of $K^{\Sigma}=\left.K\right|_{T \Sigma}$ along $\Sigma, P={ }^{\Sigma} \operatorname{tr} K^{\Sigma}$, respectively. The outward null expansion of $\Sigma$ is the quantity $\theta^{+}=P+H$ and the inward null expansion is $\theta^{-}=P-H$. The null expansions $\theta^{ \pm}$are the traces of the null second fundamental forms $\chi^{ \pm}=K^{\Sigma} \pm A$.

\section{Definition 2.1. A smooth, embedded, compact, two-sided surface $\Sigma$ is a marginally outer trapped surface (MOTS) if $\theta^{+}=0$ on $\Sigma$.}

Unless otherwise stated, we shall consider data sets $(M, g, K)$ with the following properties. We assume $M$ is a compact manifold with boundary $\partial M$ such that $\partial M=\partial^{-} M \cup$ $\partial^{+} M$ is the disjoint union of a possibly empty inner boundary $\partial^{-} M$, which we endow with the normal vector field pointing into $M$ and the non-empty outer boundary $\partial^{+} M$ which we endow with the normal vector field pointing out of $M$. We assume the outer boundary is a barrier, i.e. $\theta^{+}\left[\partial^{+} M\right]>0$. All fields are assumed to be smooth up to boundary. 
Definition 2.2. We say that $\Sigma$ bounds a region $\Omega \subset M$ with respect to $\partial^{+} M$, if the boundary $\partial \Omega$ is the disjoint union $\partial \Omega=\Sigma \cup \partial^{+} M$.

In this case, the normal pointing into $\Omega$ will be used as the outer normal for $\Sigma$.

Note that if $\Sigma$ bounds with respect to $\partial^{+} M$, then $\Sigma$ is homologous to $\partial^{+} M$.

For the existence results, Theorems 3.1 and 5.1, we need a non-empty $\partial^{-} M$ with $\theta^{+}\left[\partial^{-} M\right]<0$ as inner barrier surface. On the other hand, for the area bound, Theorem 6.5, and Theorem 7.3, which shows regularity of the trapped region, we allow $\partial^{-} M$ to be empty, and assume that $\partial^{-} M$ is a weak barrier, $\theta^{+}\left[\partial^{-} M\right] \leq 0$, if nonempty.

Definition 2.3. If $(M, g, K)$ is as before, with $\partial^{-} M$ possibly empty, then an outermost MOTS is a MOTS $\Sigma$ which bounds a region $\Omega$ with respect to $\partial^{+} M$ as in Definition 2.2 with the following properties. If $\Sigma^{\prime}$ is a MOTS bounding a set $\Omega^{\prime}$ with respect to $\partial^{+} M$ with $\Omega^{\prime} \subset \Omega$, then $\Omega^{\prime}=\Omega$.

We recall the strong maximum principle for MOTS. Note that it is only valid if two surfaces touch with the normals pointing in the same direction, as the surfaces have to be oriented the same way to use the maximum principle for quasilinear elliptic equations of second order [AG05, GT98].

Proposition 2.4. Let $(M, g, K)$ be an initial data set and let $\Sigma_{i} \subset M, i=1,2$ be two connected $C^{2}$-surfaces touching at one point $p$, such that the outer normals of $\Sigma_{i}$ agree at $p$. Assume furthermore that $\Sigma_{2}$ lies to the outside of $\Sigma_{1}$, that is in the direction of its outer normal near $p$, and that

$$
\sup _{\Sigma_{1}} \theta^{+}\left[\Sigma_{1}\right] \leq \inf _{\Sigma_{2}} \theta^{+}\left[\Sigma_{2}\right]
$$

Then $\Sigma_{1}=\Sigma_{2}$.

If $\theta^{+}\left[\partial^{-} M\right]<0$ and $\theta^{+}\left[\partial^{+} M\right]>0$ then by continuity the parallel surfaces to $\partial^{ \pm} M$, i.e. the level sets of the distance $\operatorname{dist}\left(\cdot, \partial^{ \pm} M\right)$, will satisfy the same inequality if the distance is sufficiently small. For later use we formalize this in the following definition.

Definition 2.5. Assume $\theta^{+}\left[\partial^{-} M\right]<0$ and $\theta^{+}\left[\partial^{+} M\right]>0$. Denote by $\Sigma_{s}^{ \pm}$the parallel surface to $\partial^{ \pm} M$ at distance s. Let

$$
\rho^{+}\left(M, g, K ; \partial^{+} M\right):=\sup \left\{s: \Sigma_{s}^{+} \text {is smooth, embedded and } \theta^{+}\left[\Sigma_{s}^{+}\right]>0\right\}
$$

and

$$
\rho^{-}\left(M, g, K ; \partial^{-} M\right):=\sup \left\{s: \Sigma_{s}^{-} \text {is smooth, embedded and } \theta^{+}\left[\Sigma_{s}^{-}\right]<0\right\},
$$

where we set $\rho^{-}\left(M, g, K ; \partial^{-} M\right)=\infty$ if $\partial^{-} M=\emptyset$. Let

$$
\rho(M, g, K ; \partial M):=\min \left\{\rho^{+}\left(M, g, K ; \partial^{+} M\right), \rho^{-}\left(M, g, K ; \partial^{-} M\right)\right\} .
$$

Note that $\rho(M, g, K ; \partial M)$ only depends on the geometry of $(M, g, K)$. In fact we have

Lemma 2.6. Assume $\theta^{+}\left[\partial^{-} M\right]<0$ and $\theta^{+}\left[\partial^{+} M\right]>0$. Let $\|A\|_{C^{0}(\partial M)}$ be the norm of the second fundamental form of the boundary. There is a constant $C$ depending only on $\inf _{\partial M}\left|\theta^{+}[\partial M]\right|,\|K\|_{C^{1}(M)}$, $\left\|{ }^{M} \operatorname{Rm}\right\|_{C^{0}(M)}$, and $\|A\|_{C^{0}(\partial M)}$ such that

$$
\rho(M, g, K ; \partial M)^{-1} \leq C .
$$


The significance of Definition 2.5 lies in the following lemma, which is an immediate consequence of the strong maximum principle.

Lemma 2.7. If $(M, g, K)$ is as before, with $\partial^{-} M$ possibly empty, and $\Sigma \subset M$ is a smooth MOTS homologous to $\partial^{+} M$, then

$$
\operatorname{dist}(\Sigma, \partial M) \geq \rho(M, g, K ; \partial M) .
$$

Later, we will need the injectivity radius of $(M, g)$, restricted to MOTS. By the previous lemma these surfaces cannot enter a collar neighborhood of $\partial M$ if $\partial M$ is a barrier, and thus we only need to consider the injectivity radius of points at least distance $\rho(M, g, K ; \partial M)$ away from $\partial M$.

Definition 2.8. For $p \in M$ let $\operatorname{inj}(M, g ; p)$ be the injectivity radius of $(M, g)$ at $p$. Then denote

$$
\operatorname{inj}_{\rho}(M, g, K ; \partial M):=\inf \{\operatorname{inj}(M, g ; p): \operatorname{dist}(p, \partial M) \geq \rho(M, g, K ; \partial M)\} .
$$

Let $\Sigma$ be a MOTS and let $F: \Sigma \times(\varepsilon, \varepsilon) \rightarrow M$ be a normal variation of $\Sigma$, that is $F(\cdot, 0)=\operatorname{id}_{\Sigma}$ and $\left.\frac{\partial F}{\partial s}\right|_{s=0}=f v$ for a function $f \in C^{\infty}(\Sigma)$. Then the variation of $\theta^{+}$ at $\Sigma$ is given by the operator

$$
\begin{aligned}
\left.\frac{\partial \theta^{+}[F(\Sigma, s)]}{\partial s}\right|_{s=0} & =L_{M} f \\
& =-{ }^{\Sigma} \Delta f+2 S\left({ }^{\Sigma} \nabla f\right)+f\left({ }^{\Sigma} \operatorname{div} S-\frac{1}{2}\left|\chi^{+}\right|^{2}-|S|^{2}+\frac{1}{2}{ }^{\Sigma} \mathrm{Sc}-\mu-J(v)\right) .
\end{aligned}
$$

Here ${ }^{\Sigma} \Delta,{ }^{\Sigma} \nabla$ and ${ }^{\Sigma}$ div are the Laplace-Beltrami operator, the tangential gradient and the divergence along $\Sigma$. Furthermore $S(\cdot)=K(\nu, \cdot)^{T}$, where $(\cdot)^{T}$ denotes orthogonal projection to $T \Sigma .{ }^{\Sigma} \mathrm{Sc}$ is the scalar curvature of $\Sigma, \mu=\frac{1}{2}\left({ }^{M} \mathrm{Sc}-|K|^{2}+(\operatorname{tr} K)^{2}\right)$, and $J=\operatorname{div} K-d(\operatorname{tr} K)$. This operator is not self-adjoint. However, the general theory for elliptic operators of second order implies that $L_{M}$ has a unique eigenvalue $\lambda$ with minimal real part. This eigenvalue is real, and the corresponding eigenfunction does not change sign. It is called the principal eigenvalue of $L_{M}$. In [AMS05, AMS07] the following notion was introduced:

Definition 2.9. A MOTS is called stable if the principal eigenvalue of $L_{M}$ is non-negative.

A strictly stable MOTS, that is with $\lambda>0$, can be deformed in the direction of the outer normal such that $\theta^{+}>0$ on the deformed surfaces. To see this simply use the principal eigenfunction with the positive sign as the lapse of a normal deformation. Analogously, unstable surfaces can be deformed in the direction of the outer normal such that $\theta^{+}<0$ on the deformed surface.

For a further discussion on stability see [AMS05, AMS07,AM05]. We shall need Theorem 1.2 from [AM05].

Theorem 2.10. Suppose $\Sigma$ is a stable MOTS in $(M, g, K)$ homologous to $\partial^{+} M$. Then the second fundamental form A satisfies the inequality

$$
\|A\|_{\infty} \leq C\left(\|K\|_{C^{1}(M)},\left\|^{M} \operatorname{Rm}\right\|_{C^{0}(M)}, \operatorname{inj}_{\rho}(M, g, K ; \partial M)^{-1}\right) .
$$


Note that in the reference [AM05] this theorem is proven for $M$ without boundary. The same method gives the estimate where the dependency $\operatorname{inj}(M, g)$ in the original statement is replaced by $\operatorname{inj}_{\rho}(M, g, K ; \partial M)$, as this is the quantity which needs to be controlled to apply the Hoffman-Spruck Sobolev inequality.

Subsequently we denote by $B_{r}^{M}(O)$ the open ball in $M$ with radius $r$ around $O$, and by $B_{r}^{\Sigma}(p)$ the intrinsic open ball in $\Sigma$.

Let $M$ be as above and let $\Sigma \subset M$, be a compact smooth embedded two-sided surface, and let $G_{\Sigma}$ be the normal exponential map of $\Sigma$ :

$$
G_{\Sigma}: \Sigma \times(-\operatorname{dist}(\Sigma, \partial M), \operatorname{dist}(\Sigma, \partial M)) \rightarrow M:(p, r) \mapsto \exp _{p}^{M}(r v),
$$

where $\exp _{p}^{M}: T_{p} M \rightarrow M$ is the exponential map of $M$ at $p$. Locally $G_{\Sigma}$ is injective and well behaved, this is the content of the following well-known lemma. We shall focus on the local outer injectivity in the following sense. We denote by $\operatorname{inj}(M, g ; \Sigma)$ the injectivity radius on $(M, g)$ restricted to $\Sigma$.

Lemma 2.11. If $\Sigma \subset M$ is as above with bounded curvature, there exists $0<i_{0}^{+}(\Sigma)<$ $\operatorname{inj}(M, g ; \Sigma)$, depending only on $\operatorname{inj}(M, g ; \Sigma),\left\|{ }^{M} \operatorname{Rm}\right\|_{C^{0}}$, and $\sup _{\Sigma}|A|$, such that for all $x \in \Sigma$ the map

$$
\left.G_{\Sigma}\right|_{B_{i_{0}^{+}(\Sigma)}^{\Sigma}}(x) \times\left[0, i_{0}^{+}(\Sigma)\right): B_{i_{0}^{+}(\Sigma)}^{\Sigma}(x) \times\left[0, i_{0}^{+}(\Sigma)\right) \rightarrow M
$$

is a diffeomorphism on its image, and such that the sheets

$$
\Sigma_{x, i_{0}^{+}(\Sigma)}^{s}:=G_{\Sigma}\left(B_{i_{0}^{+}(\Sigma)}^{\Sigma}(x), s\right)
$$

are discs with bounded curvature $\sup _{\Sigma^{s}}|A| \leq 2 \sup _{\Sigma}|A|$, for $s \in\left[0, i_{0}^{+}(\Sigma)\right)$.

This lemma reflects the local well-behavedness of the distance surfaces to $\Sigma$, in particular including the curvature bound. In contrast the next definition aims at the global behavior. Again, we only focus on the outward injectivity.

Definition 2.12. The outer injectivity radius of $\Sigma$ is

$$
i^{+}(\Sigma):=\sup \left\{\delta:\left.G_{\Sigma}\right|_{\Sigma \times[0, \delta)} \rightarrow M \text { is injective }\right\} .
$$

It is intuitively clear that if $i^{+}(\Sigma)$ is smaller than $i_{0}^{+}(\Sigma)$, then the surface nearly meets itself on the outside. A precise formulation is given by the following lemma.

Lemma 2.13. Let $\Sigma$ be a compact, embedded and two-sided surface with $i^{+}(\Sigma)<$ $\frac{1}{2} i_{0}^{+}(\Sigma)$. Then there exist two points $p, q \in \Sigma$ with $^{M} \operatorname{dist}(p, q)=2 i^{+}(\Sigma) b u t^{\Sigma} \operatorname{dist}(p, q) \geq$ $i_{0}^{+}(\Sigma)>2 i^{+}(\Sigma)$.

The points $p$ and $q$ can be joined by a geodesic segment $\gamma$ in $M$, which is orthogonal to $\Sigma$ at $p$ and $q$ and as a set

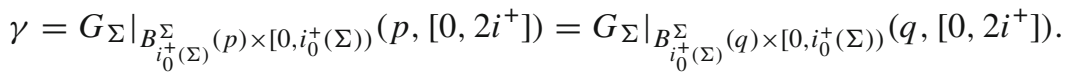

Proof. From the definition of $i^{+}$we know that

$$
G_{\Sigma}\left(\cdot, i^{+}(\Sigma)\right): \Sigma \rightarrow M
$$

is not injective. Thus there exist two points $p, q \in \Sigma$ which map to the same point $O \in M$. By Lemma $2.11^{\Sigma} \operatorname{dist}(p, q) \geq i_{0}^{+}(\Sigma)$. Furthermore $O$ has distance $i^{+}(\Sigma)$ to 


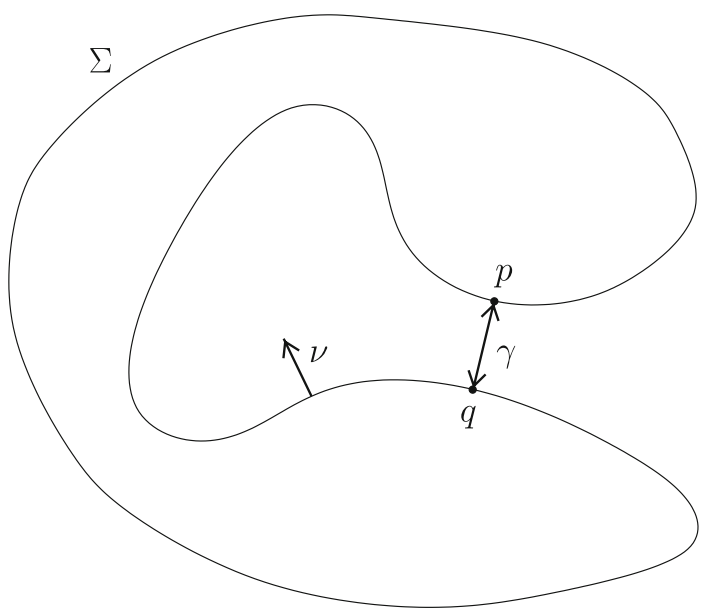

Fig. 1. A surface that nearly meets itself

$\Sigma$ and to $p, q$ so $\operatorname{dist}(O, \Sigma)=\operatorname{dist}(O, p)$ and hence the geodesic segment $\gamma_{p}$ joining $O$ to $p$ is perpendicular to $\Sigma$. Similarly the geodesic segment $\gamma_{q}$ joining $O$ and $q$ is perpendicular to $\Sigma$. Thus $\operatorname{dist}(p, q) \leq 2 i^{+}(\Sigma)$. If $\operatorname{dist}(p, q)<2 i^{+}(\Sigma)$ then there would be a parallel surface to $\Sigma$ at distance $d<i^{+}(\Sigma)$ which intersects itself, which is not possible as $G_{\Sigma}(\cdot, d)$ is injective. Thus $\operatorname{dist}(p, q)=2 d$ and $\gamma_{p}$ and $\gamma_{q}$ must form a smooth geodesic, as otherwise the angle at $O$ could be smoothed out to yield a shorter geodesic.

Figure 1 shows the situation in the lemma. It follows from the definition of $i^{+}(\Sigma)$ that the points $p, q$ minimize the distance between the sheets $B_{i_{0}^{+}(\Sigma)}^{\Sigma}(p)$ and $B_{i_{0}^{+}(\Sigma)}^{\Sigma}(q)$, and hence $\gamma$ is orthogonal to $\Sigma$ at $p$ and $q$. In addition $\gamma$ does not intersect $\Sigma$ in any other points except $p$ and $q$. If we parameterize $\gamma$ by arc length as a curve joining $p$ to $q$, the tangent to $\gamma$ at $p$ coincides with the normal $v$ to $\Sigma$. Similarly, with $\gamma$ arc length parameterized as a curve joining $q$ to $p$, the tangent to $\gamma$ at $q$ coincides with the normal $v$ to $\Sigma$ at $q$. This means that $\gamma$ lies completely on the outside of $\Sigma$.

For later reference, we need the following smoothing result from [KH97, Lemma 6].

Lemma 2.14. Let $\Sigma_{1}, \Sigma_{2} \subset M$ be smooth two-sided surfaces which intersect transversely in a smooth curve $\gamma$. Let $v_{i}$ be the outer normals of $\Sigma_{i}, i=1,2$. Choose one connected component $\Sigma^{ \pm}$of each set $\Sigma_{i} \backslash \gamma$ such that in a neighborhood of $\gamma$ the piece $\Sigma^{-}$lies in the outside of $\Sigma_{1}$ and the piece $\Sigma^{+}$in the outside of $\Sigma_{2}$. Then for any neighborhood $U$ of $\gamma$ there exists a smooth surface $\Sigma$ and a continuous and piecewise smooth bijection $\Phi: \Sigma^{+} \cup \Sigma^{-} \cup \gamma \rightarrow \Sigma$ such that

(1) $\Phi(x)=x$ for all $x \in\left(\Sigma^{+} \cup \Sigma^{-}\right) \backslash U$,

(2) $\left(\Sigma^{+} \cup \Sigma^{-}\right) \backslash U=\Sigma \backslash U$, and

(3) $\theta^{+}[\Sigma](x) \leq \theta^{+}\left[\Sigma^{+}\right](x)$ for $x \in \Sigma^{+}$and $\theta^{+}[\Sigma](x) \leq \theta^{+}\left[\Sigma^{-}\right](x)$ for $x \in \Sigma^{-}$.

Moreover $\Sigma$ lies in the connected component of $U \backslash\left(\Sigma^{+} \cup \Sigma^{-} \cup \gamma\right)$ into which the outer normals $v^{ \pm}$of $\Sigma^{ \pm}$point.

Briefly stated, this procedure works by replacing the inward corner near $\gamma$ by a smooth patch with $\theta^{+}$very negative. The reason why this procedure works is that the corner is a concentration of negative mean curvature, that is negative $\theta^{+}$. 


\section{Existence of MOTS}

This section is devoted to a proof of Schoen's existence theorem for MOTS [Sch04] in the presence of barrier surfaces.

Theorem 3.1. Let $(M, g, K)$ be a smooth, compact initial data set with $\partial M$ the disjoint union $\partial M=\partial^{-} M \cup \partial^{+} M$ such that $\partial^{ \pm} M$ are non-empty, smooth, compact surfaces without boundary and $\theta^{+}\left[\partial^{-} M\right]<0$ with respect to the normal pointing into $M$ and $\theta^{+}\left[\partial^{+} M\right]>0$ with respect to the normal pointing out of $M$. Then there exists a nonempty, smooth, embedded MOTS $\Sigma$ homologous to $\partial^{+} M$.

Remark 3.2. The proof presented here readily carries over to $n$ dimensional $M$ with $3 \leq n \leq 5$. The dimensional restriction is due to the method used for the curvature estimates in Proposition 3.3 in [SY81]. Higher dimensional replacements for this proposition are accessible via methods from geometric measure theory, cf. [Eic07].

3.1. Setup and outline. Consider $\bar{M}:=M \times \mathbf{R}$ equipped with the metric $\bar{g}=g+d z^{2}$, and define $\bar{K}$ on $\bar{M}$ as the pull-back of $K$ under the projection $\pi: M \times \mathbf{R} \rightarrow M:(p, z) \mapsto p$. For a function $f$ on $M$ we consider $N=\operatorname{graph} f:=\{(p, f(p)): p \in M\}$, with induced metric $\bar{g}$, which is of the form

$$
\bar{g}_{i j}=g_{i j}+\nabla_{i} f \nabla_{j} f, \quad \bar{g}^{i j}=g^{i j}-\frac{\nabla^{i} f \nabla^{j} f}{1+|\nabla f|^{2}} .
$$

The mean curvature of $N$ with respect to the downward normal is

$$
\mathcal{H}[f]=\operatorname{div}\left(\frac{\nabla f}{\sqrt{1+|\nabla f|^{2}}}\right) .
$$

Furthermore let

$$
\mathcal{P}[f]=\operatorname{tr}_{N} \bar{K}
$$

be the trace of $\bar{K}$ taken along $N$. Now we can write Jang's equation as

$$
\mathcal{J}[f]=\mathcal{H}[f]-\mathcal{P}[f]=0 .
$$

We shall consider the Dirichlet problem for this equation with boundary values $\left.f\right|_{\partial^{ \pm} M}=$ $\mp Z$, for constants $Z>0$.

Equation (3.1) is a quasilinear elliptic equation of divergence form. In particular, it is a prescribed mean curvature equation with gradient dependent lower order term. For such equations the strong maximum principle does not apply directly to give upper and lower bounds for the solution, without assuming extra conditions for example on the size of the domain. Further, the boundary gradient estimates needed for the proof of existence of classical solutions typically require restrictions on the geometry of the boundary. Therefore we cannot prove existence of solutions to the Dirichlet problem directly for Eq. (3.1). In general it is to be expected that solutions to the Dirichlet problem blow up in the interior.

We follow the approach of [SY81] and regularize Jang's equation by adding a capillarity term. Thus we consider instead of (3.1), the equation

$$
\mathcal{J}_{\tau}[f]=\mathcal{J}[f]-\tau f=0
$$


for $\tau>0$. After suitably modifying the data, we are able to apply Leray-Schauder theory [GT98] to prove existence of solutions to the Dirichlet problem. Letting $\tau \rightarrow 0$ gives a sequence of solutions which by uniform curvature estimates for graph $f_{\tau}$ has a subsequence which converges to a solution of Jang's equation (which in general may have blowups).

The goal is in fact to prove existence of MOTS by constructing a blowup solution to Jang's equation. For this purpose, we set $Z=\delta / \tau$ for a suitable $\delta$ and let $\tau \rightarrow 0$.

A key observation of [SY81] is that solutions to (3.2) satisfy interior estimates for the second fundamental form, uniformly in $\tau$. These estimates allow us to pick out a subsequence of solutions which converges to a blowup solution of Jang's equation. After applying a sequence of renormalizations using the fact that Jang's equation is translation invariant, we get a vertical solution, which projects to a MOTS on $M$.

The last part of the argument proceeds exactly as in [SY81], and therefore the only thing which needs to be discussed here is the Dirichlet problem.

3.2. Preparing the data. We will assume that $(M, g, K)$ is embedded into a four-dimensional Lorentz manifold $(L, h)$ such that $g$ and $K$ are the first and second fundamental forms of $M$ induced by $h$. As we do not require the dominant energy condition to hold, it is rather simple to produce an extension $(L, h)$ of $(M, g, K)$. To this end extend $g$ to $M \times \mathbf{R}$ by setting $g_{t}=g+t K$ on the slice $M \times t$. As $K$ is symmetric, so is $g_{t}$ and there exists $t_{0}>0$ such that $g_{t}$ is positive definite for $t \in\left(-t_{0}, t_{0}\right)$. Then define $h$ on $L:=M \times\left(-t_{0}, t_{0}\right)$ to be

$$
h=-d t^{2}+g_{t}
$$

This is a Lorentz metric and obviously induces $g$ as the first fundamental form on the slice $M_{0}=M \times\{0\}$. That $K$ is the second fundamental form follows from the second variation formula, which implies that the second fundamental form of $M_{0}$ is given by

$$
\left.\frac{\partial}{\partial t}\right|_{t=0} g_{t}=K .
$$

Let $t$ be a time function on $L$ with $M=\{t=0\}$ and $s^{+}(x):=\operatorname{dist}\left(x, \partial^{+} M\right)$ the distance function to $\partial^{+} M$. For small $s, t$, let $\Sigma_{s, t}^{+}$be the surface given by the intersection of the level sets of $s^{+}$and $t$. Let $n$ be the timelike normal of the $t$-level sets and let $v$ be the spacelike normal of the $s^{+}$-level sets, inside the $t$-levels, extending the outward pointing normal on $\partial^{+} M$. This defines normal fields $n, v$ at the surfaces $\Sigma_{s, t}^{+}$as well as the corresponding null normals $l^{ \pm}=n \pm v$. For small $s, t$, we have $\theta^{+}\left[\Sigma_{s, t}^{+}\right]>0$.

Now perform a Lorentz rotation of the normals $n, v$ to get

$$
\tilde{v}=\cosh \alpha \nu+\sinh \alpha n, \quad \tilde{n}=\sinh \alpha \nu+\cosh \alpha n .
$$

Let $\mathbb{I}_{a b}^{\mu}$ be the second fundamental form of the surfaces $\Sigma_{s, t}^{+}$so that $H=h^{a b}\left\langle\mathbb{I}_{a b}, v\right\rangle$ and $P=h^{a b}\left\langle\mathbb{I}_{a b}, n\right\rangle$, where $h_{a b}$ is the metric on $\Sigma_{s, t}^{+}$. Then with respect to the normals $\tilde{v}, \tilde{n}$ we have

$$
\tilde{H}=\cosh \alpha H+\sinh \alpha P, \quad \tilde{P}=\sinh \alpha H+\cosh \alpha P
$$

and the corresponding null expansions

$$
\tilde{\theta}^{ \pm}=\tilde{P} \pm \tilde{H}
$$


are given by

$$
\tilde{\theta}^{ \pm}=e^{ \pm \alpha} \theta^{ \pm}
$$

Further we note

$$
\begin{aligned}
\tilde{H} & =\frac{1}{2} e^{\alpha} \theta^{+}-\frac{1}{2} e^{-\alpha} \theta^{-}, \\
\tilde{P} & =\frac{1}{2} e^{\alpha} \theta^{+}+\frac{1}{2} e^{-\alpha} \theta^{-} .
\end{aligned}
$$

Deform $M$ to $\tilde{M}$ by bending up along the outgoing future light cone at $\partial^{+} M$. By doing so, we get the spacelike and timelike normals to agree with $\tilde{v}, \tilde{n}$ for any $\alpha$. As the deformed $\tilde{M}$ approaches the light cone, we have $\alpha \rightarrow \infty$. Therefore there is an $\alpha$ such that $\tilde{H}, \tilde{P}$ are arbitrarily close to $\frac{1}{2} e^{\alpha} \theta^{+}$. In particular, if $\theta^{+}>0$, we can achieve that both $\tilde{H}$ and $\tilde{P}$ are positive near the outer boundary of $\tilde{M}$.

We can proceed similarly at the inner boundary $\partial^{-} M$, where $\theta^{+}<0$ with respect to the inward pointing normal. This means that $\theta^{-}<0$ with respect to the outward pointing normal. Then we can proceed as above, bending along the past inward lightcone. This will result in $\tilde{H}>0, \tilde{P}<0$ (where now $\tilde{H}$ is defined with respect to the outward normal of $M$ as usual).

This constructs a deformed Cauchy data set $(\tilde{M}, \tilde{g}, \tilde{K})$. Let $\partial \tilde{M}$ be the boundary of $\tilde{M}$ constructed by bending as above. Clearly the boundary $\partial \tilde{M}$ is the union $\partial \tilde{M}=\partial^{-} \tilde{M} \cup \partial^{+} \tilde{M}$, with $\tilde{H}>0$ on $\partial \tilde{M}$ and $\tilde{P}>0$ on $\partial^{+} \tilde{M}, \tilde{P}<0$ on $\partial^{-} \tilde{M}$. Let

$$
\Sigma_{s}^{ \pm}:=\left\{x \in \tilde{M}: \operatorname{dist}\left(x, \partial^{ \pm} \tilde{M}\right)=s\right\}
$$

be the parallel surfaces to $\partial^{ \pm} \tilde{M}$ and

$$
U_{s}^{ \pm}:=\left\{x \in \tilde{M}: \operatorname{dist}\left(x, \partial^{ \pm} \tilde{M}\right)<s\right\}
$$

be the respective tubular neighborhoods. Given $\varepsilon>0$, there exists $\delta>0$ such that we can ensure the following properties:

$$
\begin{array}{ccccc}
\theta^{+}\left[\Sigma_{s}^{-}\right]<0 & \text { and } & \theta^{+}\left[\Sigma_{s}^{+}\right]>0 & \text { for } & s \in[0,4 \varepsilon], \\
H\left[\Sigma_{s}^{-}\right]>\delta & \text { and } & H\left[\Sigma_{s}^{+}\right]>\delta & \text { for } & s \in[0,2 \varepsilon], \\
P\left[\Sigma_{s}^{-}\right] \leq 0 & \text { and } & P\left[\Sigma_{s}^{+}\right] \geq 0 & \text { for } & s \in[0,2 \varepsilon],
\end{array}
$$

the data is unchanged in $M_{3 \varepsilon}$.

We abuse notation here by computing $H$ with respect to the outward pointing normal for $\partial \tilde{M}$, but compute $\theta^{+}$still with respect to the inward pointing normal near $\partial^{-} \tilde{M}$, which makes $\theta^{+}=P-H$ near $\partial^{-} \tilde{M}$.

Fix such an $\varepsilon>0$ and let $\zeta(s)$ be a non-negative cutoff function on $s \geq 0$, such that $\zeta(s)=0$ for $s \in[0, \varepsilon], \zeta(s)>0$ for $s>\varepsilon$, and $\zeta(s)=1$ for $s \geq 2 \varepsilon$. Now define $\zeta(x)=\zeta(d(x, \partial \tilde{M}))$, and consider the data set $(\tilde{g}, \zeta \tilde{K})$. From now on we denote this data set by $(M, g, K)$. The important point to note here is that this final cut-off does not affect the first property of (3.3), so that we still retain the barrier effect of the boundary. 
We find that with respect to the cut-off data we have the following properties near the boundary:

$$
\begin{aligned}
& \theta^{+}\left[\Sigma_{s}^{-}\right]<0 \text { and } \theta^{+}\left[\Sigma_{s}^{+}\right]>0 \text { for } s \in[0,4 \varepsilon], \\
& H\left[\Sigma_{s}^{-}\right]>\delta \text { and } H\left[\Sigma_{s}^{+}\right]>\delta \text { for } s \in[0,2 \varepsilon], \\
& K \equiv 0 \text { in } U_{\varepsilon} \text {, and }
\end{aligned}
$$

the data is unchanged in $M_{3 \varepsilon}$.

3.3. Existence proof. In order to construct solutions to the Dirichlet problem for (3.2), we consider, following [SY81], the family of equations

$$
\mathcal{H}[f]-\sigma \mathcal{P}[f]=\tau f,\left.\quad f\right|_{\partial M}=\sigma \phi
$$

for $\sigma \in[0,1]$ and $\tau \in[0,1]$. We need the following estimates.

Proposition 3.3. Let $N$ be the graph of a function $f$ satisfying the equation

$$
\mathcal{H}[f]-\sigma \mathcal{P}[f]=F \text { in } M
$$

with $F \in C^{1}(\bar{M})$, then the second fundamental form A of $N$ satisfies the estimate

$$
|A|(p, f(p)) \leq C\left(\left\|^{M} \operatorname{Rm}\right\|_{C^{0}},\|K\|_{C^{1}}, \operatorname{dist}(p, \partial M)^{-1}, \operatorname{inj}(M, g, p)^{-1},\|F\|_{C^{1}}\right) .
$$

In fact, if we extend the normal $\bar{v}$ of $N$ to $M \times \mathbf{R}$, then

$$
|\bar{\nabla} \bar{\nu}|(p, t) \leq C\left(\left\|{ }^{M} \operatorname{Rm}\right\|_{C^{0}},\|K\|_{C^{1}}, \operatorname{dist}_{M}(p, \partial M)^{-1}, \operatorname{inj}(M, g, p)^{-1},\|F\|_{C^{1}}\right) .
$$

Proof. This is analogous to [SY81, Prop. 1 and Prop. 2].

Proposition 3.4. Let $f_{\sigma, \tau}$ be a solution to (3.5) with parameters $\Sigma$ and $\tau$. Then $f_{\sigma, \tau}$ satisfies the estimates

$$
\sup _{M}\left|f_{\sigma, \tau}\right| \leq \max \left\{3\|K\|_{C^{0}} / \tau, \sup _{\partial M}|\phi|\right\},
$$

and

$$
\sup _{M}\left|\nabla f_{\sigma, \tau}\right| \leq \max \left\{c\left(\left\|{ }^{M} \mathrm{Rm}\right\|_{C^{0}}+\|\nabla K\|_{C^{0}}\right) / \tau, \sup _{\partial M}\left|\nabla f_{\sigma, \tau}\right|\right\} .
$$

Proof. This follows from the maximum principle, as in [SY81, Sect. 4].

Hence we can estimate the gradient once we have a boundary gradient estimate.

Proposition 3.5. Let $(M, g, K)$ be a data set such that there are $\varepsilon>0, \delta>0$, such that for $s \in[0, \varepsilon]$ the surfaces

$$
\Sigma_{s}:=\{p \in M: \operatorname{dist}(p, \partial M)=s\}
$$

satisfy $H>\delta$. Further, assume that $K \equiv 0$ in $\{p: \operatorname{dist}(p, \partial M)<\varepsilon\}$. Let $f_{\tau, \sigma}$ be a solution of

$$
\mathcal{J}_{\tau, \sigma}\left[f_{\tau, \sigma}\right]=\mathcal{H}\left[f_{\tau, \sigma}\right]-\sigma \mathcal{P}\left[f_{\tau, \sigma}\right]-\tau f_{\tau, \sigma}=0,
$$


such that $f_{\tau, \sigma}$ is constant on each component of $\partial M$. Suppose that

$$
\sup _{M}\left|f_{\tau, \sigma}\right|=m<\infty \text { and } \sup _{\partial M}\left|f_{\tau, \sigma}\right| \leq \frac{\delta}{2 \tau} .
$$

Then

$$
\sup _{\partial M}\left|\nabla f_{\tau, \sigma}\right| \leq \max \left\{\frac{1}{\sqrt{3}}, 2 \varepsilon^{-1} m\right\}
$$

Proof. We proceed by constructing a barrier near $\partial^{-} M$. Consider functions $w$ of the form

$$
w=\psi(s), \quad s=\operatorname{dist}\left(\cdot, \partial^{-} M\right),
$$

where $\psi:[0, \varepsilon] \rightarrow \mathbf{R}$ is a scalar function. For functions of this form we have

$$
\mathcal{J}_{\tau, \sigma}[w]=-\frac{\psi^{\prime}}{\left(1+\left(\psi^{\prime}\right)^{2}\right)^{1 / 2}} H\left[\Sigma_{s}\right]+\frac{\psi^{\prime \prime}}{\left(1+\left(\psi^{\prime}\right)^{2}\right)^{1 / 2}}-\tau \psi
$$

in the neighborhood where $K \equiv 0$. To construct an upper barrier near one component $\Sigma$ of $\partial^{-} M$, set $w^{+}:=\psi^{+}(s)$ with $\psi^{+}(s)=a+b s$, where $a$ is the value of $f_{\tau, \sigma}$ on $\Sigma$. We can then pick $b$ so large that $\frac{b}{\left(1+b^{2}\right)^{1 / 2}} \geq \frac{1}{2}$, that is $b \geq \frac{1}{\sqrt{3}}$. Then (3.6) yields that

$$
\begin{aligned}
\mathcal{J}_{\tau, \sigma}\left[w^{+}\right] & \leq-\frac{\delta}{2}+\tau|a|-\tau b s \\
& \leq-\frac{\delta}{2}+\tau \sup _{\partial M}|f|-\tau b s \leq-\tau b s \leq 0 .
\end{aligned}
$$

We can then choose $b$ so large that $a+b \varepsilon \geq m$, that is $b \geq 2 \varepsilon^{-1} m$. Thus we have constructed an upper barrier, the construction of the lower barrier is analogous.

The barrier near $\partial^{+} M$ can be constructed analogously, using the expression

$$
\mathcal{J}_{\tau, \sigma}[w]=\frac{\psi^{\prime}}{\left(1+\left(\psi^{\prime}\right)^{2}\right)^{1 / 2}} H\left[\Sigma_{s}\right]+\frac{\psi^{\prime \prime}}{\left(1+\left(\psi^{\prime}\right)^{2}\right)^{1 / 2}}-\tau \psi
$$

for $\mathcal{J}_{\tau, \sigma}$ near $\partial^{+} M$.

As a corollary, we find that given suitable boundary data, Eq. (3.5) is uniformly elliptic, where the ellipticity constant does not depend on $\sigma \in[0,1]$. Thus we conclude that there exists a solution to (3.5) with $\sigma=1$ and $\tau>0$ for such data by applying Leray-Schauder theory.

Corollary 3.6. Let $(M, g, K)$ and $\phi \in C^{\infty}(\partial M)$ be as in Proposition 3.5. Then the equation

$$
\left\{\begin{array}{l}
\mathcal{H}\left[f_{\tau}\right]-\mathcal{P}\left[f_{\tau}\right]=\tau f_{\tau} \\
\left.f_{\tau}\right|_{\partial M}=\phi
\end{array}\right.
$$

has a solution $f_{\tau}$ in $C^{2, \alpha}(\bar{M})$ with

$$
\left\|f_{\tau}\right\|_{C^{2, \alpha}(\bar{M})} \leq C / \tau,
$$

where the constant $C=C\left(\left\|{ }^{M} \mathrm{Rm}\right\|_{C^{0, \alpha}},\|K\|_{C^{1, \alpha}, \varepsilon^{-1}}\right)$. 
Proof. This is analogous to [SY81, Lemma 3].

We now specify the precise data on $\partial M$. Set

$$
\phi=\left\{\begin{array}{rll}
\frac{\delta}{2 \tau} & \text { on } & \partial^{-} M \\
-\frac{\delta}{2 \tau} & \text { on } & \partial^{+} M
\end{array},\right.
$$

where $\delta$ is as in Proposition 3.5. We then solve (3.8) with this data to obtain a family of functions $f_{\tau}$. Note that the gradient estimate forces $f_{\tau}$ to be uniformly large near the boundary. Denote $M_{\varepsilon}=\{p \in M: \operatorname{dist}(p, \partial M)>\varepsilon\}$.

Lemma 3.7. There exists an $\varepsilon^{\prime}>0$ such that the functions $f_{\tau}$ satisfy

$$
\left|f_{\tau}\right| \geq \frac{\delta}{4 \tau} \quad \text { in } \quad M \backslash M_{\mathcal{E}^{\prime}} .
$$

As in [SY81, Sect. 4] we can now use the curvature estimate from Proposition 3.3 to obtain a limit for graph $f_{\tau}$ as $\tau \rightarrow 0$. By the previous lemma we can restrict ourselves to $M_{\mathcal{E}^{\prime}}$ away from the boundary, as $f_{\tau} \rightarrow \infty$ uniformly on $M \backslash M_{\mathcal{E}^{\prime}}$. This gives the following result.

Proposition 3.8. There exists a sequence $\tau_{i} \rightarrow 0$ such that graph $f_{\tau_{i}}$ in $M_{\varepsilon^{\prime}}$ converges to a smooth manifold $N_{0}$ satisfying $H+P=0 . N_{0}$ consists of a disjoint collection of components, which are either graphs or cylinders over compact surfaces $\Sigma$.

Let $\Omega_{ \pm}:=\left\{p: f_{\tau_{i}}(p) \rightarrow \pm \infty\right\}$ and $\Omega^{0}:=\left\{p: \sup _{i \geq 1}\left|f_{\tau_{i}}(p)\right|<\infty\right\}$. Then $M$ is $a$ disjoint union $M=\Omega^{0} \cup \Omega^{+} \cup \Omega^{-}$. The set $\Sigma:=\partial \Omega^{-} \backslash \partial^{+} M$ consists of marginally trapped surfaces with $\theta^{+}=0$ with respect to the normal pointing into $\Omega^{-}$.

The fact that $\Sigma$ satisfies $\theta^{+}=0$, can be seen as follows. Since the $f_{\tau_{i}}$ converge to $-\infty$ in $\Omega^{-}$and are bounded below outside of $\Omega^{-}$, there are just two possibilities for the convergence of $N_{\tau_{i}}=$ graph $f_{\tau_{i}}$ to $N_{0}$ near each component $\Sigma^{\prime}$ of $\Sigma$. The first possibility is that $\Sigma^{\prime}$ is the interface between $\Omega^{+}$and $\Omega^{-}$. Then $N_{0}$ has a cylindrical component $\Sigma^{\prime} \times \mathbf{R}$, and the convergence is such that the downward normal $\bar{\nu}_{\tau}$ of $N_{\tau_{i}}$ converges to the normal of $\Sigma^{\prime}$ pointing out of $\Omega^{-}$. As $N_{0}$ satisfies $\mathcal{H}\left[N_{0}\right]-\mathcal{P}\left[N_{0}\right]=0$ with respect to the limit of $\bar{v}_{\tau_{i}}$, this implies that $H-P=0$ on $\Sigma^{\prime}$ with respect to the outward pointing normal, and hence $\theta^{+}=P+H=0$ with respect to the inward pointing normal as claimed. The second possibility is that $\Sigma^{\prime}$ is an interface between $\Omega^{0}$ and $\Omega^{-}$. Then near $\Sigma^{\prime}, N_{0}$ is a graph over $\Omega^{0}$ which asymptotes to $\Sigma^{\prime} \times \mathbf{R}$, and since $f_{\tau_{i}} \rightarrow-\infty$ in $\Omega^{-}$, this graph goes to $-\infty$ near $\Sigma^{\prime}$ as well. Again we can conclude that $\bar{\nu}_{\tau_{i}}$ converges to the normal of $N_{0}$ pointing out of $\Omega^{-}$. Furthermore, $\mathcal{H}-\mathcal{P}=0$ on $\Sigma^{\prime} \times \mathbf{R}$ with respect to this normal, as it is the limit of $N_{0}$, which satisfies $\mathcal{H}-\mathcal{P}=0$. Hence we again conclude that $\theta^{+}\left[\Sigma^{\prime}\right]=0$.

From Lemma 3.7 we know that $\Omega^{+}$contains a neighborhood of $\partial^{-} M$ and $\Omega^{-}$contains a neighborhood of $\partial^{+} M$, so neither one of them is trivial. In particular $\partial \Omega^{-}$is the disjoint union $\partial \Omega^{-}=\Sigma \cup \partial^{+} M$, where $\Sigma \subset M$ is contained in the interior of $M$.

Recall that we had to modify the data for the existence proof. We now show that $\Sigma$ can not enter the region where we modified the data. To see this, note that a neighborhood of $\partial^{-} M$ is foliated by surfaces $\Sigma_{s}^{-}$with $\theta^{+}\left[\Sigma_{s}^{-}\right]<0$. If $\Sigma$ enters this region there is a minimal $s$, with $\Sigma_{s}^{-} \cap \Sigma \neq \emptyset$. This surface touches $\Sigma$ with their outward normals pointing in the same direction. Thus, by the strong maximum principle, $\Sigma=\Sigma_{s}^{-}$, a contradiction. Furthermore, there is a neighborhood of $\partial^{+} M$ foliated by surfaces $\Sigma_{s}^{+}$ with $\theta^{+}\left[\Sigma_{s}^{+}\right]>0$. We can then proceed analogously to get a contradiction to $\Sigma$ entering 
this neighborhood. As data set is modified only in the neighborhoods discussed above, we find that $\Sigma$ lies entirely in the region where the data is unchanged.

We thus conclude the proof of Theorem 3.1 by finding our solution $\Sigma$ in the unmodified region of $(M, g, K)$.

It is an interesting possibility that the existence theory developed here for the Dirichlet problem for Jang's equation can be used to generalize Yau's result in [Yau01, Theorem 5.2] to more general boundary geometries. This possibility will be investigated by the authors in future work.

\section{Blowup Surfaces are Stable}

While not actually necessary for the main result of the paper, we present an extension of the results of Sect. 3. From the arguments in [SY81] it is clear that $\Sigma$ has only components which are symmetrized stable, where symmetrized stable refers to non-negativity of the operator (cf. [GS06])

$$
\tilde{L}_{M} f=-{ }^{\Sigma} \Delta f+f\left(\frac{1}{2}^{\Sigma} \mathrm{Sc}-\frac{1}{2}|\chi|^{2}-\mu-J(v)\right) .
$$

Here we want to show that they are in fact stable in the sense of MOTS.

Theorem 4.1. The surface $\Sigma$ constructed in the proof of Theorem 3.1 is a stable MOTS.

Remark 4.2. By the same argument we can prove that any blow-up surface obtained by the capillarity term regularization of Jang's equation is a stable surface, in particular those in [SY81]. Note that all of these surfaces are MOTS provided one chooses the right orientation of the normal.

Proof. The stability of $\Sigma$ will follow from a barrier argument. Assume that $\Gamma$ is an unstable component of $\Sigma$. We will show that in this case the functions $f_{\tau_{i}}$ are bounded below $+\infty$ in a neighborhood of $\Gamma$. Hence $\Gamma$ lies in the interior of $\Omega^{+} \cup \Omega^{0}$ and can not be part of $\partial \Omega^{-}$, which contradicts the assumption that $\Gamma$ is a component of $\Sigma$.

If $\Gamma$ is unstable, let $\phi>0$ be a suitably scaled eigenfunction to the principal eigenvalue. We can extend the vector field $\phi v$ to a neighborhood of $\Gamma$, and flow $\Gamma$ by this vector field. This yields a map $F: \Gamma \times[-1,1] \rightarrow M$ and constant $\Lambda>0$ with the following properties. We will denote $\Gamma_{s}=F(\Gamma, s)$.

1. $\Gamma_{0}=\Gamma$.

2. $\Gamma_{s} \subset \Omega^{+}$if $s \in[-1,0)$ and $\Gamma_{s} \cap \Omega^{+}=\emptyset$ if $s \in(0,1]$.

3. $\frac{\partial F}{\partial s}=\beta v$, where $v$ is the normal to $\Gamma_{s}$ extending the outward pointing normal $v$ on $\Gamma$, and $\beta$ satisfies the estimates

$$
\Lambda^{-1} \leq \beta \leq \Lambda, \quad \text { and } \quad\left|\frac{\partial \beta}{\partial s}\right| \leq \Lambda
$$

4. Outside of $\Omega^{+}$we have $\theta^{+}\left[\Gamma_{s}\right]<0$ and inside $\theta^{+}\left[\Gamma_{s}\right]>0$ and

$$
\Lambda^{-1} s \leq\left|\theta^{+}\left[\Gamma_{s}\right]\right| \leq \Lambda s \quad \text { for all } \quad s \in[-1,1] .
$$

5. We can assume that $\|K\|_{C^{0}(M)} \leq \Lambda$. 
For an interval $\left(s_{1}, s_{2}\right) \subset[-1,1]$ we denote by $A\left(s_{1}, s_{2}\right)$ the annular region $F\left(\Gamma \times\left(s_{1}, s_{2}\right)\right)$, which is foliated by the $\Gamma_{s}$ for $s \in\left(s_{1}, s_{2}\right)$ and has boundary $\partial A\left(s_{1}, s_{2}\right)=\Gamma_{s_{1}} \cup \Gamma_{s_{2}}$.

We will construct a subsolution $w$ of Jang's equation, satisfying $\mathcal{J}[w] \geq \eta>0$. The function $w$ will be constant on the $\Gamma_{s}$, that is $w=\phi(s)$. We will later use the positivity of $\eta$ to infer that $w+m_{\tau}$ are in fact subsolutions for $\mathcal{J}_{\tau}$, where $m_{\tau}$ is a suitably chosen constant.

Lemma 4.3. For $w=\phi(s)$ we can compute Jang's operator to be the following expression:

$$
\mathcal{J}[w]=\frac{\phi^{\prime}}{\beta \sigma} \theta^{+}-\left(1+\frac{\phi^{\prime}}{\beta \sigma}\right) P-\sigma^{-2} K(v, v)+\frac{\phi^{\prime \prime}}{\beta^{2} \sigma^{3}}-\frac{\phi^{\prime}}{\beta^{3} \sigma^{3}} \frac{\partial \beta}{\partial s} .
$$

Here $\sigma^{2}=1+\beta^{-2} \phi^{\prime 2}$.

To construct $w$ we will proceed in three steps, which amount to constructing $w$ on the annuli $A_{1}:=A(-\delta, 0), A_{2}:=A(0, \varepsilon)$, and $A_{3}:=A(\varepsilon, 2 \varepsilon)$, where $\delta$ and $\varepsilon$ will be fixed during the construction.

We start with the construction of $\phi$ in $A_{2}=A(0, \varepsilon)$, which will fix $\varepsilon$, but not quite $\phi$. In this region all we know is that $\theta^{+}\left[\Gamma_{s}\right] \leq 0$, so we make the assumption $\phi^{\prime} \leq-\mu<0$, where we will fix $\mu$ in the course of the argument. This renders the first term in (4.1) to be non-negative. We can thus estimate that

$$
\mathcal{J}[w] \geq-\frac{c_{1}}{\mu^{2}}+c_{2} \frac{\phi^{\prime \prime}}{\left|\phi^{\prime}\right|^{3}},
$$

for constants $c_{1}, c_{2}>0$ depending only on $\Lambda$, provided we choose $\mu \geq \Lambda$. To see this, note that $\sigma$ is comparable to $\left|\phi^{\prime}\right|$ provided the latter is bounded away from zero. The fact that the term containing $P$ in (4.1) is of the form $c_{1} / \mu^{2}$ follows from the Taylor expansion of the square root. To get that the right hand side of (4.2) is positive we must satisfy

$$
\frac{\phi_{2}^{\prime \prime}}{\left|\phi^{\prime}\right|^{3}} \geq \frac{c_{0}}{\mu^{2}}
$$

where $c_{0}=\frac{c_{1}+1}{c_{2}}+1$ is a positive constant depending only on $\Lambda$. We will later use $c_{0}>1$ and $c_{0} c_{2}>1$.

We make the following ansatz for $\phi$ in $[0, \varepsilon]$ :

$$
\phi_{2}(s)=a_{2}\left(1+\frac{s}{\varepsilon}\right)^{2 / 3}+b_{2}
$$

for constants $a_{2}, b_{2}$ to be determined. We compute that

$$
\begin{aligned}
& \phi_{2}^{\prime}(s)=\frac{2 a_{2}}{3 \varepsilon}\left(1+\frac{s}{\varepsilon}\right)^{-1 / 3}, \\
& \phi_{2}^{\prime \prime}(s)=-\frac{2 a_{2}}{9 \varepsilon^{2}}\left(1+\frac{s}{\varepsilon}\right)^{-4 / 3}=-\frac{9 \varepsilon^{2}}{8 a_{2}^{3}} \phi_{2}^{\prime}(s)^{4} .
\end{aligned}
$$

As we want to have $\phi_{2}^{\prime}<0$, we must choose $a_{2}<0$ which renders $\phi_{2}^{\prime \prime}(s)>0$. So in order to get $\phi^{\prime}(s) \leq-\mu$ it is sufficient to take

$$
-\mu=\phi_{2}^{\prime}(\varepsilon)=\frac{a_{2}}{3 \varepsilon} 2^{2 / 3},
$$


as $\left|\phi^{\prime}\right|$ is increasing. This implies

$$
a_{2}^{2}=2^{-4 / 3} 9 \varepsilon^{2} \mu^{2} .
$$

To satisfy (4.3), we require that

$$
\frac{c_{0}}{\mu^{2}} \leq \frac{\phi^{\prime \prime}(\varepsilon)}{\left|\phi^{\prime}(\varepsilon)\right|^{3}}=\frac{9 \varepsilon^{2}}{8 a_{2}^{3}} \phi^{\prime}(\varepsilon)=\frac{3 \varepsilon}{a_{2}^{2}} 2^{-7 / 3} .
$$

This is equivalent to

$$
a_{2}^{2} \leq \frac{3 \varepsilon \mu^{2}}{c_{0}} 2^{-7 / 3} .
$$

Combining with (4.7) we find the condition

$$
9 \varepsilon^{2} \mu^{2} 2^{-4 / 3} \leq \frac{3 \varepsilon \mu^{2}}{c_{0}} 2^{-7 / 3}
$$

or

$$
\varepsilon \leq \frac{1}{6 c_{0}}
$$

Thus we choose $\varepsilon=\frac{1}{6 c_{0}}$. Note that since $c_{0}>1, \varepsilon<\frac{1}{6}<\frac{1}{2}$. Modulo fixing $\mu$ and the vertical shift, we are done with $\phi$ on $(0, \varepsilon)$. Note that $\varepsilon$ does not depend on $\mu$ which is important in view of the fact that we will later choose $\mu$ as a function of $\varepsilon$. Note further that $\mathcal{J}[w] \geq \frac{1}{\mu^{2}}$ on $A_{2}$ by construction.

For $A_{3}:=A(\varepsilon, 2 \varepsilon)$ we will make the ansatz $w=\phi_{3}(s)$, with $s \in[\varepsilon, 2 \varepsilon)$. As we are in the region $s>\varepsilon$, where $\varepsilon$ has been fixed by the construction in $A_{2}$, we have $\theta^{+} \leq-\Lambda^{-1} \varepsilon$ and thus the first term in (4.1) is estimated by $\kappa:=\frac{\varepsilon}{\sqrt{2} \Lambda}>0$ from below. We can estimate the whole expression as follows:

$$
\mathcal{J}[w] \geq \kappa-\frac{c_{1}}{\mu^{2}}-c_{2} \frac{\left|\phi_{3}^{\prime \prime}(s)\right|}{\left|\phi_{3}^{\prime}(s)\right|^{3}},
$$

where we again assumed $\left|\phi^{\prime}(s)\right| \geq \mu \geq \Lambda$, and $c_{1}$ and $c_{2}$ are constants depending only on $\Lambda$. We can ensure that the second term is small, that is

$$
\frac{c_{1}}{\mu^{2}} \leq \frac{\kappa}{4}
$$

provided

$$
\mu^{2} \geq \frac{4 c_{1}}{\kappa}
$$

It remains to find a function, which allows us to choose $\mu$ large while keeping the term

$$
c_{2} \frac{\left|\phi_{3}^{\prime \prime}(s)\right|}{\left|\phi_{3}^{\prime}(s)\right|^{3}}<\frac{\kappa}{4} .
$$


We make the ansatz

$$
\phi_{3}(s)=a_{3} \log \left(1-\frac{s-\varepsilon}{\varepsilon}\right)+b_{3}
$$

and compute

$$
\begin{aligned}
& \phi_{3}^{\prime}(s)=-\frac{a_{3}}{\varepsilon}\left(1-\frac{s-\varepsilon}{\varepsilon}\right)^{-1}, \\
& \phi_{3}^{\prime \prime}(s)=-\frac{a_{3}}{\varepsilon^{2}}\left(1-\frac{s-\varepsilon}{\varepsilon}\right)^{-2} .
\end{aligned}
$$

As we need $\phi_{3}^{\prime}(\varepsilon)=-\mu$, to be able to fit $\phi_{3}$ to $\phi_{2}$, we compute $-\mu=\phi_{3}^{\prime}(\varepsilon)=-\frac{a_{3}}{\varepsilon}$ or $a_{3}=\varepsilon \mu>0$. Hence $\phi_{3}^{\prime \prime}(s)<0$ and $\phi_{3}^{\prime}(s) \leq \mu$ for $s \in(\varepsilon, 2 \varepsilon)$, as desired. We still have to fix $\mu$. The goal is to simultaneously satisfy (4.11) and (4.12). Compute

$$
\frac{\left|\phi_{3}^{\prime \prime}(s)\right|}{\left|\phi_{3}^{\prime}(s)\right|^{3}}=\frac{1}{\mu^{2} \varepsilon}\left(1-\frac{s-\varepsilon}{\varepsilon}\right) \leq \frac{1}{\mu^{2} \varepsilon} .
$$

Thus we can ensure (4.12) provided $\mu^{2} \geq \frac{4 c_{2}}{\varepsilon \kappa}$. We choose

$$
\mu=\max \left\{\sqrt{\frac{4 c_{1}}{\kappa}}, \sqrt{\frac{4 c_{2}}{\varepsilon \kappa}}, \Lambda\right\},
$$

and are done constructing $\phi_{3}$ up to fixing $b_{3}$ in such a way to ensure $\phi_{2}(\varepsilon)=\phi_{3}(\varepsilon)$. Note that we have that $\phi_{3}(s) \rightarrow-\infty$ as $s \rightarrow 2 \varepsilon$, which is the desired behavior. Furthermore we have $\mathcal{J}[w] \geq \frac{\kappa}{2}>0$ in $A_{3}$.

In the region $A_{1}=A(-\delta, 0)$, where $0<\delta<1$ will be chosen later, we set $w(s)=$ $\phi_{1}(s)$. Then we estimate from (4.1) that

$$
J[w] \geq-c_{3}+c_{4} \frac{\phi_{1}^{\prime \prime}(s)}{\left|\phi_{1}^{\prime}(s)\right|^{3}},
$$

where $c_{3}$, and $c_{4}>0$ are again constants depending only on $\Lambda$. Here we assumed that $\left|\phi_{1}^{\prime}(s)\right| \geq \Lambda$ as before. The only chance to get the right hand side of this expression positive is to take $\phi_{1}(s)$ to be a function with

$$
\frac{\phi_{1}^{\prime \prime}(s)}{\left|\phi_{1}^{\prime}(s)\right|^{3}} \geq \frac{c_{3}+1}{c_{4}}:=c_{5} .
$$

We make the ansatz

$$
\phi_{1}(s)=a_{1}\left(1+\frac{s}{2 \delta}\right)^{1 / 2}+b_{1},
$$

and compute

$$
\begin{aligned}
& \phi_{1}^{\prime}(s)=\frac{a_{1}}{4 \delta}\left(1+\frac{s}{2 \delta}\right)^{-1 / 2}, \\
& \phi_{1}^{\prime \prime}(s)=-\frac{a_{1}}{16 \delta^{2}}\left(1+\frac{s}{2 \delta}\right)^{-3 / 2} .
\end{aligned}
$$


We fix $b_{1}$ such that $\phi_{1}(-\delta)=0$. This then fixes $b_{2}$ and $b_{3}$ by the requirement that $w$ is continuous on $A(-\delta, 2 \varepsilon)$. From the requirement $\phi_{1}^{\prime}(0)=\phi_{2}^{\prime}(0)=:-\mu^{\prime}$, we infer that

$$
a_{1}=-4 \mu^{\prime} \delta \text {. }
$$

Recall that $-\mu^{\prime}$ is fixed and can not be chosen freely. From $\phi_{1}^{\prime \prime}(s)>0$ we find that $\left|\phi_{1}^{\prime}(s)\right| \geq\left|\phi_{1}^{\prime}(0)\right|=\mu^{\prime}=2^{1 / 3} \mu \geq \mu=\left|\phi_{2}(\varepsilon)\right| \geq \Lambda$, so $\phi_{1}^{\prime}$ is automatically large enough to justify (4.14). To get positivity of the right hand side of (4.14) we need that

$$
c_{5} \leq \frac{\phi^{\prime \prime}(s)}{\left|\phi^{\prime}(s)\right|^{3}}=\frac{4 \delta}{a_{1}^{2}} .
$$

Solving for $a_{1}^{2}$ yields the condition

$$
a_{1}^{2} \leq \frac{4 \delta}{c_{5}} .
$$

As we already fixed $a_{1}$ in (4.15), we infer the condition

$$
\delta \leq \frac{1}{4 c_{5} \mu^{\prime 2}}
$$

So we fix $\delta=\frac{1}{4 c_{5} \mu^{\prime 2}}$ and are done. Note that $\mathcal{J}[w] \geq 1$ by construction.

To summarize, we have constructed a function $w$ on $A(-\delta, 2 \varepsilon)$ with the following properties:

(i) $w$ is $C^{1,1}$ up to the boundary in every $A(-\delta, s)$ with $s \in(-\delta, 2 \varepsilon)$. Hence $w \in W^{2, \infty} \cap C^{1,1}$ away from $\Gamma_{2 \varepsilon}$

(ii) there exists $\eta>0$ such that $J[w] \geq \eta$,

(iii) $w \equiv 0$ on $\Gamma_{-\delta}, w \leq 0$ on $A(-\delta, 2 \varepsilon)$,

(iv) there exists $C_{1}<\infty$ such that $0 \geq w \geq-C_{1}$ in $A(-\delta, \varepsilon)$, and

(v) $\left.w\right|_{\Gamma_{s}} \rightarrow-\infty$ as $s \rightarrow 2 \varepsilon$.

Here $\eta$ and $C_{1}$ are constants that only depend on $\Lambda$, as do $\delta$ and $\varepsilon$.

With this subsolution $w$, we can get a lower bound of the functions $f_{\tau}$ solving $\mathcal{J}\left[f_{\tau}\right]=\tau f_{\tau}$ near $\Gamma$ as follows. Set

$$
m:=\min \left\{\inf _{\Gamma_{-\delta}} f_{\tau}, \frac{\eta}{\tau}\right\},
$$

and consider the function

$$
w_{m}:=w+m .
$$

The goal is to apply the comparison principle for the quasilinear operator $\mathcal{J}$ to show that $w_{m} \leq f_{\tau}$ in $A(-\delta, 2 \varepsilon)$. To this end let $U$ be the region where $f_{\tau} \leq m$. From the equation we conclude that

$$
\mathcal{J}\left[f_{\tau}\right]=\tau f_{\tau} \leq \tau m \leq \eta
$$

in $U$, and moreover $f_{\tau}=m$ on $\partial U$. As $f_{\tau} \geq-\frac{C}{\tau}$ is bounded below as in Proposition 3.4, we can choose $\bar{s} \in(\varepsilon, 2 \varepsilon)$ such that $\left.w_{m}\right|_{\Gamma_{\bar{s}}} \leq \inf _{M} f_{\tau}$. 
Set $V:=U \cap A(-\delta, \bar{s})$. Then, as $\partial V \subset \partial U \cup \Gamma_{-\delta} \cup \Gamma_{\bar{s}}$, we find that $w_{m} \leq f_{\tau}$ on $\partial V$. An application of the comparison principle [GT98, Chap. 10] allows us to conclude that $w_{m} \leq f_{\tau}$ in $V$ and thus

$$
w_{m} \leq f_{\tau} \quad \text { in } \quad A(-\delta, 2 \varepsilon)
$$

By construction, there is a constant $C_{1}$ such that $w+C_{1} \geq 0$ in $A(-\delta, \epsilon)$ and hence

$$
m-C_{1} \leq w_{m} \text { in } A(-\delta, \varepsilon) \text {. }
$$

Thus we infer the estimate

$$
f_{\tau} \geq \min \left\{\inf _{\Gamma_{-\delta}} f_{\tau}, \frac{\eta}{\tau}\right\}-C_{1} \quad \text { in } A(-\delta, \varepsilon) .
$$

We can now conclude the argument. Take the sequence $\tau_{i}$ and the functions $f_{\tau_{i}}$ from Proposition 3.8. By construction $f_{\tau_{i}}$ is uniformly bounded below on $\Gamma_{-\delta}$ as $\Gamma_{-\delta}$ is compactly contained in $\Omega^{+} \cup \Omega^{0}$, hence as $\tau_{i} \rightarrow 0$ the term on the right hand side of (4.18) is bounded below as $\tau_{i} \rightarrow 0$. Thus $A(-\delta, \varepsilon) \subset \Omega^{+} \cup \Omega^{0}$, which is a contradiction, since we assumed that $\Gamma \subset A(-\delta, \varepsilon)$ was a boundary component of $\partial \Omega^{-}$.

This concludes the proof of Theorem 4.1.

\section{Weak Barriers}

In this section we will slightly improve Theorem 3.1 to allow interior boundaries where we just have the weak inequality $\theta^{+}\left[\partial^{-} M\right] \leq 0$, instead of the strict inequality assumed in Theorem 3.1 .

Theorem 5.1. Let $(M, g, K)$ be a smooth, compact initial data set with $\partial M$ the disjoint union $\partial M=\partial^{-} M \cup \partial^{+} M$ such that $\partial^{ \pm} M$ are non-empty, smooth, compact surfaces without boundary and $\theta^{+}\left[\partial^{-} M\right] \leq 0$ with respect to the normal pointing into $M$ and $\theta^{+}\left[\partial^{+} M\right]>0$ with respect to the normal pointing out of $M$.

Then there exists a smooth, embedded, stable MOTS $\Sigma \subset M$ homologous to $\partial^{+} M$. $\Sigma$ may have components which agree with components of $\partial^{-} M$ that satisfy $\theta^{+}=0$.

In this case we can not use the strong maximum principle to exclude that $\Sigma$ touches $\partial^{-} M$ as in Lemma 2.7. For the proof of Theorem 5.1 we shall need the following lemma.

Lemma 5.2. Let $\Sigma$ be a connected, two-sided, compact, embedded surface with $\theta^{+} \leq 0$ and $\theta^{+} \not \equiv 0$. Then for every $\varepsilon>0$ there exists a smooth, embedded surface $\Sigma^{\prime}$ in the $\varepsilon$-neighborhood of $\Sigma$, which lies to the outside of $\Sigma$ but does not touch $\Sigma$, is a graph over $\Sigma$, and satisfies $\theta^{+}<0$.

Proof. Consider the following equation for a function $F: \Sigma \times[0, \bar{s}) \rightarrow M$ :

$$
\left\{\begin{array}{l}
\frac{d F}{d s}=-\theta^{+} v \\
F(\cdot, 0)=\operatorname{id}_{\Sigma}
\end{array}\right.
$$

Here, $v$ is the outer normal as usual. This is a weakly parabolic equation for $F$, in fact it is a generalization of the mean curvature flow. To see this, recall that $\theta^{+}=H+P$, 
where $H$ is the mean curvature, and $P={ }^{M} \operatorname{tr} K-K(v, v)$ is a term only depending on first derivatives of $F$. Thus the flow in Eq. (5.1) is

$$
\frac{d F}{d s}=-H v-\text { lower order. }
$$

Hence it has the same symbol as the mean curvature flow and thus is a quasilinear parabolic equation.

The theory of parabolic equations guarantees the existence of a solution for a small time interval $[0, \bar{s})$, see for example [HP99, Sect. 7]. Furthermore, any surface $\Sigma_{s}=$ $F(\Sigma, s)$ for $s \in(0, \bar{s})$ is smooth. From a standard argument using the strong maximum principle we conclude that $\theta^{+}<0$ instantly. To see this, recall that the evolution equation for $\theta^{+}$has the form

$$
\frac{\partial \theta^{+}}{\partial s}=-L_{s} \theta^{+}=\Delta \theta^{+}-2 S\left(\nabla \theta^{+}\right)-\theta^{+} Q,
$$

where $L_{S}$ is the linearization of $\theta^{+}$along $\Sigma_{s}$, with

$$
Q={ }^{\Sigma} \operatorname{div} S-\frac{1}{2}\left|\chi^{+}\right|^{2}-|S|^{2}+\frac{1}{2}^{\Sigma} \mathrm{Sc}-\mu+J(\nu)-\frac{1}{2}\left(\theta^{+}\right)^{2}+\theta^{+} \operatorname{tr} K,
$$

where all geometric quantities are computed on $\Sigma_{s}$. Note that $L_{s}$ equals $L_{M}$ on MOTS. By smoothness we have that $Q$ is bounded for a short time, whence we can choose

$$
a>\max _{s \in[0, \bar{s} / 2], x \in \Sigma_{s}}|Q(x, s)| .
$$

Let $u=e^{-a s} \theta^{+}$and compute

$$
\left(\frac{\partial}{\partial s}-\Delta\right) u=-2 S(\nabla u)-(Q+a) u .
$$

The coefficient of the zeroth order term is negative. Hence the strong maximum principle from [Lie96] is applicable to $u$ and implies that $u$ instantly becomes negative, implying that $\theta^{+}$instantly becomes negative.

If $s$ is small enough, $\Sigma_{s}$ will also be embedded. As $\theta^{+} \leq 0$, the flow (5.1) moves the surface in the direction of $v$ everywhere, and hence outward, in particular $\Sigma_{s} \cap \Sigma=\emptyset$. As the initial speed is given by $\left|\theta^{+}\right|$, which is bounded, the surfaces $\Sigma_{s}$ will be arbitrarily close to $\Sigma$, as long as $s>0$ is small enough. Hence we can choose $\Sigma^{\prime}$ to be one of the $\Sigma_{s}$.

Proof of Theorem 5.1. The main difficulty here is that $\partial^{-} M$ may have multiple connected components $\partial^{-} M=\Gamma_{1} \cup \ldots \cup \Gamma_{N}$ where some of the $\Gamma_{k}$ satisfy $\theta^{+}=0$, to which we can not apply Lemma 5.2 directly.

Lemma 5.2 allows us to flow the boundary components $\Gamma_{k}$ with $\theta^{+} \leq 0$ and $\theta^{+} \not \equiv 0$ in the direction of their outer normal $v$, that is into $M$, to replace $M$ by a manifold $M_{1}$ which is such that $\partial^{-} M_{1}$ is still embedded and each component of $\partial^{-} M_{1}$ either has $\theta^{+}<0$ or $\theta^{+}=0$. As the boundary components with $\theta^{+}=0$ do not allow the application of Theorem 3.1, we have to tweak them a little.

Pick one such component $\Gamma$ of $\partial^{-} M$ with $\theta^{+}[\Gamma]=0$, then there are three cases. Either, as a MOTS, $\Gamma$ is not stable, $\Gamma$ is stable, but not strictly stable, or $\Gamma$ is strictly stable.

When $\Gamma$ is not stable, let $\phi>0$ be an eigenfunction for the principal eigenvalue $\lambda<0$ for the operator $L_{M}$ on $\Gamma$. Extend the vector field $\phi v$ to a neighborhood of $\Gamma$ and 
flow $\Gamma$ for a short time interval along this vector field. This yields a foliation $\left\{\Gamma_{s}\right\}_{s \in[0, \varepsilon)}$ of a neighborhood of $\Gamma$, such that $\Gamma_{0}=\Gamma$ and $\Gamma_{s}$ lies inside of $M$ and has $\theta^{+}<0$ when $s>0$. Hence, we push $\Gamma$ a little inward and obtain a strictly trapped surface.

In the other two cases we need to flow the components with respect to the vector field $-\phi v$, where $\phi>0$ is again the principal eigenfunction of $L_{M}$ on $\Gamma$. So we have to assume that there is an extension $\left(M^{\prime}, g^{\prime}, K^{\prime}\right)$ of $(M, g, K)$ with $M \subset M^{\prime}, g=\left.g^{\prime}\right|_{M}$ and $K=\left.K^{\prime}\right|_{M}$ such that $\partial^{-} M$ lies in the interior of $M^{\prime}$. Such an extension can be constructed by simply gluing $[0,1] \times \partial^{-} M$ to $M$ along $\partial^{-} M$ and smoothly extending $g$ and $K$ to the added piece. Keeping this in mind, we can now move the other boundary components $\Gamma$ inwards in the following way.

If $\Gamma$ is strictly stable, then by flowing in the direction $-\phi v$, we construct a foliation $\left\{\Gamma_{s}\right\}_{s \in(-\varepsilon, 0]}$ of a neighborhood of $\Gamma$, such that $\Gamma_{0}=\Gamma$ and $\Gamma_{s}$ lies in the direction $-v$, that is outside of $M$ and has $\theta^{+}<0$ if $s<0$. We choose one of the $\Gamma_{s}$ as a new inner boundary. We will later use the fact that the region between the former boundary $\Gamma$ and the new boundary $\Gamma_{S}$ is foliated by surfaces with $\theta^{+}<0$ to ensure that the constructed MOTS does not enter this region.

The last case is where $\Gamma$ is stable but not strictly stable. In this case we also flow $\Gamma$ in the direction $-\phi v$ and construct a foliation $\left\{\Gamma_{s}\right\}_{s \in(-\varepsilon, 0]}$ of a neighborhood of $\Gamma$, such that $\Gamma_{0}=\Gamma$ and $\Gamma_{s}$ lies outside of $M$ and

$$
\left.\frac{\partial}{\partial s}\right|_{s=0} \theta^{+}\left[\Gamma_{s}\right]=0 .
$$

We will change the data $K^{\prime}$ along the surfaces $\Gamma_{s}$ by replacing $K^{\prime}$ by

$$
\tilde{K}=K^{\prime}-\frac{1}{2} \psi(s) h_{s},
$$

where $h_{s}$ is the metric on $\Gamma_{s}$ and $\psi: \mathbf{R} \rightarrow \mathbf{R}$ is a $C^{1}$ function with $\psi(s)=0$ for $s>0$. Note that $\tilde{\theta}^{+}\left[\Gamma_{s}\right]$, which means the quantity $\theta^{+}$computed with respect to the new data $\left(M^{\prime}, g^{\prime}, \tilde{K}\right)$, satisfies

$$
\tilde{\theta}^{+}\left[\Gamma_{s}\right]=\theta^{+}\left[\Gamma_{s}\right]-\psi(s) .
$$

As $\theta^{+}\left[\Gamma_{s}\right]$ vanishes to first order in $s$ at $s=0$ by (5.2), we can extend $\psi$ as a $C^{1,1}$ function to $\tilde{M}$ such that $\theta^{+}<0$ on all $\Gamma_{s}$, if $s<0$ is close enough to zero. Hence, this case is similar to the strictly stable case. It is clear that we can choose $\Gamma_{s}$ in such a way that $\|\tilde{K}\|_{C^{1}(\tilde{M})} \leq 2\|K\|_{C^{1}(M)}$.

In summary, by this construction we have replaced $(M, g, K)$ by a manifold $(\tilde{M}, \tilde{g}, \tilde{K})$ which are both embedded in a data set $\left(M^{\prime}, g^{\prime}, K^{\prime}\right)$. The outer boundaries of $M$ and $\tilde{M}$ agree and have $\theta^{+}>0$, while the inner boundary of $\tilde{M}$ has $\theta^{+}\left[\partial^{-} \tilde{M}\right]<0$. The data $\tilde{K}$ is $C^{1,1}$ in $\tilde{M}$.

The set $U:=M \backslash \tilde{M} \subset M^{\prime}$, corresponding to the boundary components we moved out of $M$, is foliated by surfaces $\Sigma_{S}$ with $\theta^{+}\left[\Sigma_{s}\right]<0$ with respect to the data $(\tilde{g}, \tilde{K})$.

We can now invoke Theorem 3.1 to find a smooth, embedded, stable MOTS $\Sigma$ in $\tilde{M}$, which bounds with respect to $\partial^{-} \tilde{M}$. Note that it is only necessary to assume $K \in C^{1, \alpha}$ for some $0<\alpha \leq 1$ for the theorem to apply. If one of the components $\Sigma^{\prime}$ of $\Sigma$ enters $U$, say the component $U^{\prime}$ of $U$, then let $\bar{s}:=\min \left\{s: \Sigma_{s} \cap \Sigma_{k} \neq \emptyset\right\}$, where the $\Sigma_{s}$ constitute the foliation of $U^{\prime}$ by strictly trapped surfaces, as above. At the point where the minimum is assumed, the outward normals of $\Sigma^{\prime}$ and $\Sigma_{\bar{s}}$ point into the same direction, and hence the strong maximum principle implies that $\Sigma_{k}=\Sigma_{\bar{s}}$, a contradiction. Thus $\Sigma \cap U=\emptyset$, 


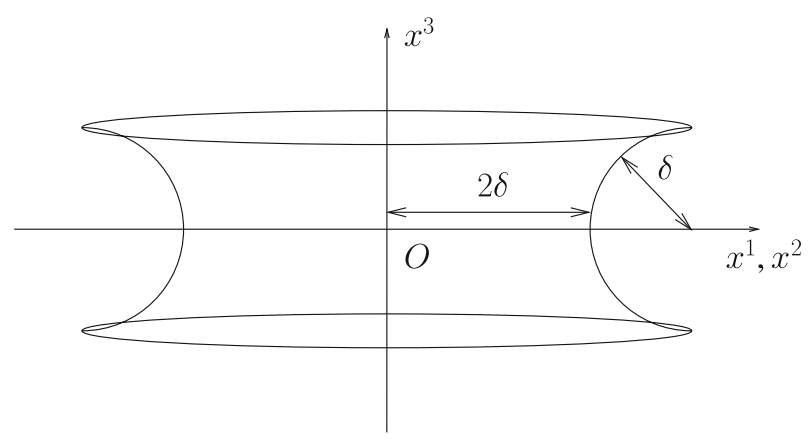

Fig. 2. The $\delta$-standard neck

and $\Sigma \subset M$ is the desired solution. Note that some components of $\Sigma$ might agree with components of $\partial^{-} M$ which have $\theta^{+}=0$.

The assertion that $\Sigma$ is stable then follows from Theorem 4.1.

As an immediate consequence of Theorem 5.1, we infer the following corollary.

Corollary 5.3. Let $(M, g, K)$ be such that $\partial M$ is the disjoint union $\partial M=\partial^{-} M \cup \partial^{+} M$, where $\partial^{+} M$ is non-empty with $\theta^{+}\left[\partial^{+} M\right]>0$ and $\partial^{-} M$ is possibly empty. If $\Sigma$ is an outermost MOTS homologous to $\partial^{+} M$, then there do not exist outer trapped surfaces enclosing $\Sigma$. In particular, $\Sigma$ is a stable MOTS.

\section{Surgery}

In this section we describe a surgery procedure to construct an outer trapped surface outside of a MOTS $\Sigma$ with small $i^{+}(\Sigma)$ and bounded curvature. In view of the existence part in Theorem 5.1, we infer a lower bound on $i^{+}(\Sigma)$ for outermost MOTS. This implies an area estimate.

Moreover, the surgery procedure guarantees that a fixed amount of the volume outside of $\Sigma$ is consumed. By iterating the surgery procedure and application of Theorem 5.1, we then infer that after a finite number of steps we arrive at a MOTS $\Sigma^{\prime}$ outside of $\Sigma$ with a lower bound on $i^{+}\left(\Sigma^{\prime}\right)$.

As usual, we assume that $\Sigma$ is homologous to $\partial^{+} M$ and denote the region bounded by $\Sigma$ and $\partial^{+} M$, that is the outside of $\Sigma$, by $\Omega$.

6.1. Neck geometry. The surgery procedure works by inserting necks with negative $\theta^{+}$. We start by constructing a suitable neck in Euclidean space, and transfer it to the geometry of $M$ in normal coordinates. Let $\delta>0$ and consider the map

$$
F:[0,2 \pi] \times\left[-\frac{\pi}{2}, \frac{\pi}{2}\right] \rightarrow \mathbf{R}^{3}:(\phi, \theta) \mapsto\left(\begin{array}{c}
\delta \sin \phi(3-\cos \theta) \\
\delta \cos \phi(3-\cos \theta) \\
\delta \sin \theta
\end{array}\right)
$$

The image of $F$ is shown in Fig. 2; we will call it the $\delta$-standard neck. Denote by the interior $I_{\delta}$ of the neck the points $\left(x^{1}, x^{2}, x^{3}\right)$ with $x^{3} \in(-\delta, \delta), x^{3}=\delta \sin \theta$ and

$$
\left(x^{1}\right)^{2}+\left(x^{2}\right)^{2} \leq \delta^{2}\left(3-\cos \theta^{2}\right) \text {. }
$$




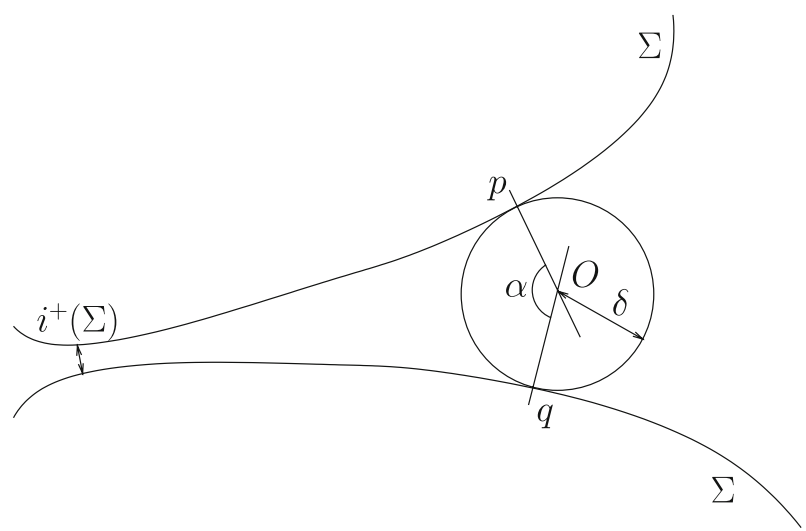

Fig. 3. Selecting the points $p$ and $q$ where a ball $B_{\delta}(O)$ touches $\Sigma$

Clearly, the open ball $B_{\delta}^{\mathbf{R}^{3}}(0)$ is contained in $I_{\delta}$.

The Euclidean mean curvature of the standard neck with respect to the normal pointing out of $I_{\delta}$ is

$$
H^{e}=-\delta^{-1}\left(1-(3-\cos \theta)^{-1} \cos \theta\right) \leq-(2 \delta)^{-1}
$$

Thus the Euclidean mean curvature of the $\delta$-standard neck can be arbitrarily negative if $\delta$ is chosen small enough. Let $r_{0}$ be such that at any point $O \in M$ with $\operatorname{dist}(O, \partial M) \geq$ $\rho(M, g, K ; \partial M) / 2$ we have geodesic normal coordinates $\left\{x^{i}\right\}$ such that for $r \leq r_{0}$ we have

$$
r^{-2}\left|g_{i j}-\delta_{i j}\right|+r^{-1}\left|\partial_{k} g_{i j}\right|+\left|\partial_{k} \partial_{l} g_{i j}\right| \leq C
$$

where $r$ is the Euclidean distance in $x$-coordinates. Then, the image of the standard neck in these coordinates will have $H<-(4 \delta)^{-1}$ if $\delta<r_{0}$ is small enough. Thus, choosing $\delta^{-1}$ large compared to $\|K\|_{C^{0}(M)}$, we can ensure that the $\delta$-standard neck has $\theta^{+}<0$.

6.2. Point selection. The goal is to consume a fixed amount of volume by application of the surgery. To this end, we have to insert a neck with $\delta$ bounded away from zero in terms of the geometry of $M$. Hence, it is not sufficient to do surgery at the points $p, q$ which realize $i^{+}(\Sigma)$. Instead, we have to find points $p, q$ such that there is a point $O$ with $\operatorname{dist}(O, \partial M) \geq \rho(M, g, K ; \partial M) / 2$ such that $B_{\delta}^{M}(O)$ touches $\Sigma$ at $p$ and $q$, and the angle of the segments joining $O$ to $p$ and $q$ at $O$ is close to $\pi$.

These points $p, q, O$ can be found as follows. Let $r_{0}$ be as above. There exist $r_{1}<r_{0}$ and $C>0$ depending only on $\left\|{ }^{M} \mathrm{Rm}\right\|_{C^{0}}$, such that $\partial B_{r}^{M}(O)$ has a second fundamental form $A_{r} \geq \frac{C}{r} \gamma_{r}$ where $\gamma_{r}$ is the induced metric on $\partial B_{r}^{M}(O)$ (use the Hessian comparison theorem for the distance function to $O$ [SY94]). Furthermore, there exists $0<r_{2}<r_{1} / 2$, depending additionally on $\sup _{\Sigma}|A|$ with the following property. If $O$ and $r<r_{2}$ are such that $\partial B_{r}^{M}(O)$ touches $\Sigma$ at $p$, then the $\Sigma$-ball $B_{r_{2}}^{\Sigma}(p)$ does not intersect the interior of $B_{r}^{M}(O)$. The important point to note is that the radius of the $\Sigma$-ball does not depend on $r$. 
Now fix $r<r_{2}$ and consider the set $U_{r} \subset \Sigma$ of points which can be touched by a ball which lies completely outside of $\Sigma$, that is,

$$
U_{r}:=\left\{p \in \Sigma: \exists O \in \Omega \text { s.t. } B_{r}^{M}(O) \subset \Omega \text { and } p \in \partial B_{r}^{M}(O)\right\} .
$$

Clearly $U_{r}$ is non-empty if $2 r<\operatorname{dist}\left(\Sigma, \partial^{+} M\right)$, as then the point $p_{1} \in \Sigma$ which realizes $\operatorname{dist}\left(\Sigma, \partial^{+} M\right)$ is in $U_{r}$. Let $\Sigma_{1}$ be the component of $\Sigma$ containing $p_{1}$. If $\Sigma_{1} \subset U_{r}$, then dist $\left(\Sigma_{1}, \Sigma \backslash \Sigma_{1}\right) \geq 2 r$. We then select $p_{2} \in \Sigma \backslash \Sigma_{1}$ such that $p_{2}$ realizes the distance $\operatorname{dist}\left(\Sigma \backslash \Sigma_{1}, \partial^{+} M \cup \Sigma_{1}\right)$, clearly $p_{2} \in U_{r}$. We can continue this process until either we found a component $\Sigma_{k}$ of $\Sigma$ with $\Sigma_{k} \not \subset U_{r}$ and $U_{r} \cap \Sigma_{k} \neq \emptyset$, or we showed that $\Sigma=U_{r}$. However, the latter can not happen if $i^{+}(\Sigma)<r$, as the points $p, q$ from Lemma 2.13 are not in $U_{r}$. Thus, there is a component $\Sigma_{k}$ of $\Sigma$ which contains a point $p \in \partial U_{r}$, the boundary of $U_{r}$ relative to $\Sigma$.

As $U_{r}$ is closed in $\Sigma$, there exists $O \in \Omega$ such that $p \in \partial B_{r}^{M}(O)$ and $B_{r}^{M}(O) \subset \Omega$. We claim that there exists $q \in \Sigma \cap \partial B_{r}^{M}(O), q \neq p$. This $q$ can be constructed as follows. Choose a sequence of points $p_{k} \in \Sigma \backslash U_{r}$ with $p_{k} \rightarrow p$. Consider the geodesic normal to $\Sigma$ emanating from $p_{k}$ outward. Let $O_{k}$ be the point at distance $r$ from $p_{k}$ on this geodesic. As $p_{k}$ is not in $U_{r}$, the ball $B_{r}\left(O_{k}\right)$ intersects $\Sigma$ in a point $q_{k}$ with $\operatorname{dist}\left(q_{k}, O_{k}\right)<r$ and $\operatorname{dist}_{\Sigma}\left(p_{k}, q_{k}\right) \geq r_{2}$, by our choice of $r$. By compactness we can assume that the $q_{k}$ converge to $q$ with $\operatorname{dist}(q, O) \leq r$ and $\operatorname{dist}_{\Sigma}(p, q) \geq r_{2}$. As $p \in U_{r}$, the open ball $B_{r}^{M}(O)$ does not intersect $\Sigma$ and thus $\operatorname{dist}(q, O)=r$.

Thus we find that, if $r<r_{2}$ and $i^{+}(\Sigma)<r$, there exist points $p \neq q \in \Sigma$ and $O \in \Omega$ such that $p, q \in \partial B_{r}(O)$. Denote the geodesic segment joining $O$ and $p$ by $\gamma_{p}$ and the segment joining $O$ and $q$ by $\gamma_{q}$. We now want to show that the angle between $\gamma_{p}$ and $\gamma_{q}$ at $O$ is close to $\pi$ if $r$ is small enough.

Consider geodesic normal coordinates around $O$. Then the segments $\gamma_{p}$ and $\gamma_{q}$ are straight lines emanating from $O$. Let $L_{p}$ be the plane orthogonal to $\gamma_{p}$ through $p$. As the curvature of $\Sigma$ is bounded, $B_{r_{3}}^{\Sigma}(p)$ is the graph of a function $u_{p}$ over $L_{p}$ with

$$
r^{-2} u_{p}+r^{-1}\left|\partial_{k} u_{p}\right|+\left|\partial_{k} \partial_{l} u_{p}\right| \leq C
$$

for $r<r_{3}$, where $r_{3}>0$ and $C<\infty$ depend only on $\operatorname{inj}_{\rho}(M, g, K ; \partial M)^{-1},\left\|{ }^{M} \operatorname{Rm}\right\|_{C^{0}(M)}$ and $\left.\sup _{\Sigma}|A|\right)$. In particular, $B_{r_{3}}^{\Sigma}(p)$ is contained in a small tubular neighborhood of $L_{p}$. Similarly, $B_{r_{3}}^{\Sigma}(q)$ is contained in a neighborhood of $L_{q}$.

Let $\alpha$ be the angle of $\gamma_{p}$ and $\gamma_{q}$ at $O$. We claim that for each $\eta>0$ there exists $r>0$ such that $|\alpha-\pi|<\eta$. Otherwise, if $\alpha$ is not close to $\pi$, the planes $L_{p}$ and $L_{q}$ intersect at distance $d$ with $d=\frac{r}{\cos (\alpha / 2)} \leq \frac{r}{\varepsilon}$. Thus, choosing $r$ small enough, we can make $L_{p}$ and $L_{q}$ intersect within $d \leq r_{3} / 2$. This implies that $B_{r_{3}}^{\Sigma}(p)$ and $B_{r_{3}}^{\Sigma}(q)$ must also intersect. This is a contradiction, as $\Sigma$ is assumed to be embedded.

6.3. Surgery. With the previous preparations, we can carry out the surgery procedure. We choose $r$ so small that the above considerations apply, giving the following properties:

1. The $(2 \delta)$-standard neck in normal coordinates around any point $O \in M$ with $\operatorname{dist}(O, \partial M)>\operatorname{inj}_{\rho}(M, g, K ; \partial M)$ has $\theta^{+}<0$ in $(M, g, K)$.

2. The $M$-ball $B_{\delta}^{M}(O)$ is contained in the interior of the image of the $(2 \delta)$-standard neck.

3. If $i^{+}(\Sigma)<\delta$, then there exist points $p, q \in \Sigma$ and $O \in \Omega$ such that $B_{\delta}(O) \subset \Omega$ and $p, q \in \partial B_{\delta}(O)$.

4. The angle $\alpha$ of $\gamma_{p}$ and $\gamma_{q}$ at $O$ satisfies $|1 / \cos \alpha+6 \tan \alpha| \leq 3 / 2$. 


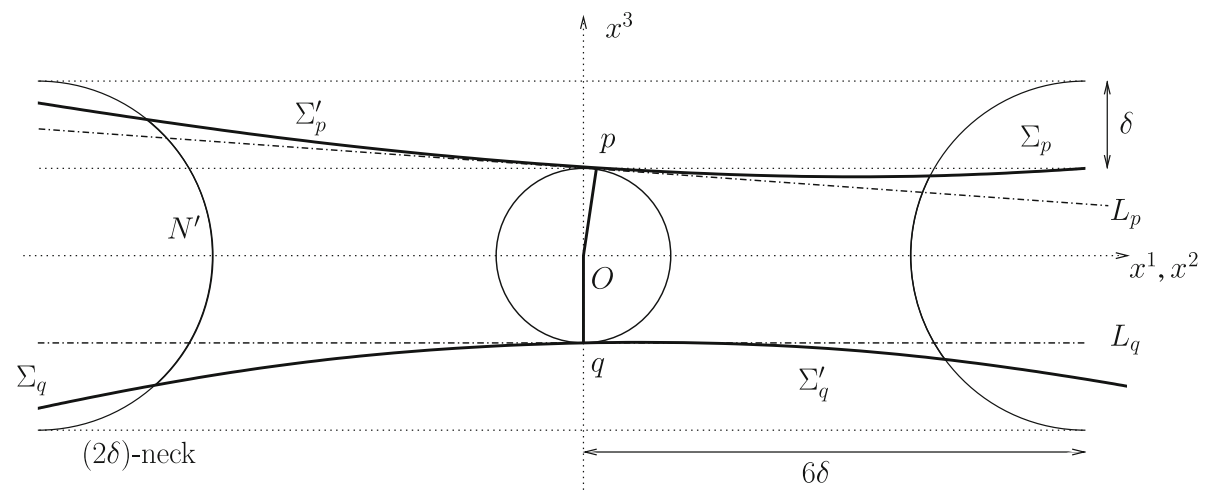

Fig. 4. The surgery in geodesic normal coordinates

Now assume that $i^{+}(\Sigma)<\delta$ and pick $p, q, O$ as in Condition 3 above, and consider geodesic normal coordinates around $O$ such that $\gamma_{q}$ lies on the negative $x^{3}$-axis. Let $N$ be the image of the $(2 \delta)$-neck centered at $O$ with its axis aligned with the $x^{3}$-coordinate axis, as in Fig. 4. Condition 4 on $\alpha$ implies that the plane $L_{p}$ is such that

$$
\left.L_{p} \cap\left\{\left(x^{1}\right)^{2}+\left(x^{2}\right)^{2} \leq 6 \delta\right\} \subset\left\{-\frac{3}{2} \delta \leq x^{3} \leq \frac{3}{2} \delta\right]\right\} .
$$

Recall that the component $\Sigma_{p}$ of $\Sigma \cap\left\{-2 \delta \leq x^{3} \leq 2 \delta\right\}$ containing $p$ is the graph over $L_{p}$ of a function $u_{p}$ with

$$
r^{-2} u_{p}+r^{-1}\left|\partial_{k} u_{p}\right|+\left|\partial_{k} \partial_{l} u_{p}\right| \leq C,
$$

where $C$ is as in Eq. (6.1). Thus, we can choose $\delta$, depending only on $C$ so small, that first $\Sigma_{p} \subset\left\{-2 \delta \leq x^{3} \leq 2 \delta\right\}$, and second $\Sigma_{p}$ and $N$ intersect transversely (note that the angle of $\Sigma$ and $L_{p}$ is of order $\delta$, whereas the angle between the neck and $L_{p}$ is uniformly bounded away from zero). We can similarly argue for $\Sigma_{q}$, so that we find that Fig. 4 is indeed accurate.

The surgery can now be performed as follows. Let $\Sigma_{p}^{\prime}$ be the component of $\Sigma \backslash N$ that contains $p$ and $\Sigma_{q}^{\prime}$ be the component that contains $q$. Let $N^{\prime}$ be the component of $N \backslash \Sigma$ between $\Sigma_{p}$ and $\Sigma_{q}$. Construct a non-smooth surface $\Sigma_{N}$ by removing $\Sigma_{p}^{\prime}$ and $\Sigma_{q}$ and adding $N^{\prime}$. By construction this surface is homologous to $\Sigma$, and hence to $\partial^{+} M$. By Condition 1, we find that the inserted neck has $\theta^{+}<0$. Condition 2 implies that $B_{\delta}(O)$ is indeed contained in the neck we added. Furthermore, at the corner $\Sigma \cap N^{\prime}$, the normals $v_{N}$ of $N^{\prime}$ and $v$ of $\Sigma$ enclose an angle $<\pi$.

We proceed by using Lemma 2.14 to smooth out this corner, thereby constructing a surface $\Sigma^{\prime}$. This $\Sigma^{\prime}$ lies outside of $\Sigma_{N}$, and agrees with $\Sigma_{N}$ except in an arbitrarily small neighborhood of the corner and has $\theta^{+} \leq 0$ and $\theta^{+} \not \equiv 0$. Note that in particular, the component of $\Sigma^{\prime}$, which contains part of $N^{\prime}$ has $\theta^{+}<0$ somewhere.

6.4. Results. By the previous surgery procedure we arrive at the following proposition:

Proposition 6.1. Let $(M, g, K)$ be a data set such that $\partial M$ is the disjoint union $\partial M=$ $\partial^{+} M \cup \partial^{-} M$ of smooth compact surfaces without boundary. Assume that $\theta^{+}\left(\partial^{+} M\right)>0$ and if $\partial^{-} M$ is non-empty, that $\theta^{+}\left(\partial^{-} M\right)<0$. 
There exists $\delta>0$ depending only on $\operatorname{inj}_{\rho}(M, g, K ; \partial M)^{-1},\left\|{ }^{M} \mathrm{Rm}\right\|_{C^{0}}$ and $\|K\|_{C^{1}}$ with the following property. If $\Sigma \subset M$ is a stable MOTS, homologous to $\partial^{+} M$, bounding $\Omega$ together with $\partial^{+} M$, and $i^{+}(\Sigma)<\delta$, then there exists a MOTS $\Sigma^{\prime}$ outside of $\Sigma$, homologous to $\partial^{+} M$ and bounding $\Omega^{\prime}$ together with $\partial^{+} M$ such that

$$
\operatorname{Vol}\left(\Omega^{\prime}\right) \leq \operatorname{Vol}(\Omega)-v_{0},
$$

where $0<v_{0}:=\inf \left\{\operatorname{Vol} B_{\delta}^{M}(p): \operatorname{dist}(p, \partial M) \geq \delta\right\}$.

Proof. The fact that $\Sigma$ is stable yields a curvature bound in view of Theorem 2.10. Then the above surgery procedure can be applied to construct $\Sigma^{\prime}$.

An immediate corollary of the above proposition is the following.

Corollary 6.2. Let $(M, g, K)$ and $\delta$ be as in Proposition 6.1. If $\Sigma$ is an outermost MOTS in $M$, then $i^{+}(\Sigma) \geq \delta$.

Proof. If $i^{+}(\Sigma)<\delta$, then Proposition 6.1, guarantees the existence of a barrier surface outside of $\Sigma$, and Theorem 5.1 implies the existence of a MOTS outside of $\Sigma$. Thus $\Sigma$ is not outermost.

More importantly, as already indicated, the fact that a surgery takes away a uniform amount of volume, gives a finiteness result, which allows us to prove the following theorem.

Theorem 6.3. Let $(M, g, K)$ be a data set such that $\partial M$ is the disjoint union $\partial M=$ $\partial^{+} M \cup \partial^{-} M$ of smooth compact surfaces without boundary. Assume that $\theta^{+}\left(\partial^{+} M\right)>0$ and if $\partial^{-} M$ is non-empty, that $\theta^{+}\left(\partial^{-} M\right)<0$. Let $\delta$ be as in Proposition 6.1.

If $\Sigma \subset M$ is a MOTS homologous to $\partial^{+} M$, then there exists a stable MOTS $\Sigma^{\prime}$, with

$$
i^{+}\left(\Sigma^{\prime}\right) \geq \delta
$$

such that $\Sigma^{\prime}$ does not intersect the region bounded by $\Sigma$ (and $\partial^{-} M$ if non-empty).

Proof. If $\Sigma$ is not stable we use Theorem 5.1 with inner boundary $\Sigma$ to construct a stable MOTS $\Sigma_{1}$ outside of $\Sigma$. If $i^{+}\left(\Sigma_{1}\right)<\delta$, then Proposition 6.1 applies and yields a barrier outside of $\Sigma_{1}$ which can be fed into Theorem 5.1 to construct a stable MOTS $\Sigma_{2}$ outside of $\Sigma_{1}$. The region bounded by $\Sigma_{1}$ and $\Sigma_{2}$ has volume bounded below by $v_{0}$, where $v_{0}$ is from Proposition 6.1. If $i^{+}\left(\Sigma_{2}\right)<\delta$, we can iterate. As each step consumes at least volume $v_{0}$ outside of $\Sigma$, this procedure must end after a finite number of steps with a surface $\Sigma_{k}$ with $i^{+}\left(\Sigma_{k}\right) \geq \delta$.

A lower bound on $i^{+}(\Sigma)$ can be used to estimate the area of $\Sigma$. This area estimate is crucial to get the compactness of the class of stable MOTS with $i^{+}(\Sigma)$ bounded below.

Proposition 6.4. Let $(M, g)$ be a compact Riemannian manifold with boundary, and $\Sigma \subset M$ an embedded, two-sided surface with bounded curvature $|A| \leq C$. Let

$$
\delta:=\min \left\{i_{0}^{+}(\Sigma), i^{+}(\Sigma)\right\} .
$$

Then there exists an absolute constant $c$ such that the following area estimate holds:

$$
|\Sigma| \leq c\left(\delta^{-1}+\sup _{\Sigma}|A|\right) \operatorname{Vol}(M) \text {. }
$$


Proof. Let $v$ be the outward pointing normal to the surfaces $\Sigma^{s}:=G_{\Sigma}(\Sigma, s)$ for $s \in[0, \delta]$, where $G_{\Sigma}$ is as in Eq. (2.1). Then ${ }^{M} \operatorname{div}(v)=H^{s}$, where $H^{s}$ denotes the mean curvature of $\Sigma^{s}$. As $\delta \leq i_{0}^{+}(\Sigma)$, the estimate

$$
\left|{ }^{M} \operatorname{div} \nu\right| \leq 2 \sup _{\Sigma^{s}}|A| \leq 4 \sup _{\Sigma}|A|
$$

follows from the definition of $i_{0}^{+}(\Sigma)$ (which has the bound on $\sup _{\Sigma^{s}}|A|$ built in).

Let $\phi$ be a cut-off function with $\phi(s)=1$ for $s \leq \delta / 4, \phi=0$ for $s \geq \delta / 2$ and $0 \leq \phi^{\prime}(s) \leq 8 \delta^{-1}$. Using the divergence theorem for the vector field $N=-\phi(s) v$ in the volume $U:=G(\Sigma,[0, \delta))$, we infer that

$$
|\Sigma|=\int_{\Sigma}\langle N, v\rangle \mathrm{d} \mu=\int_{U} M_{\operatorname{div}} N \leq \operatorname{Vol}(U)|\operatorname{div} N| .
$$

This yields the desired area estimate.

As outermost MOTS are stable, and thus have bounded curvature, we can combine this proposition with Corollary 5.3 to infer the following area bound for outermost MOTS.

Theorem 6.5. Let $(M, g, K)$ be a smooth, compact initial data set with $\partial M$ the disjoint union $\partial M=\partial^{-} M \cup \partial^{+} M$, where $\partial^{+} M$ is non-empty and has $\theta^{+}\left[\partial^{+} M\right]>0$, and $\theta^{-}\left[\partial^{-} M\right]<0$ if $\partial^{-} M$ is non-empty. Then, if $\Sigma$ is an outermost MOTS, we have the estimate

$$
|\Sigma| \leq C
$$

where $C$ depends only on $\left\|^{M} \mathrm{Rm}\right\|_{C^{0}(M)},\|K\|_{C^{1}(M)}, \operatorname{inj}_{\rho}(M, g, K, \partial M)^{-1}$, and $\operatorname{Vol}(M)$.

As the proof of the previous theorem does not assume that $\Sigma$ is connected, it also implies a bound on the number of components of an outermost MOTS.

Corollary 6.6. Let $(M, g, K)$ be as above. Then there exists a constant $N$, depending only on $\left\|^{M} \operatorname{Rm}\right\|_{C^{0}(M)},\|K\|_{C^{1}(M)}$, $\operatorname{inj}_{\rho}(M, g, K ; \partial M)^{-1}$, and $\operatorname{Vol}(M)$ such that any outermost MOTS has at most $N$ components.

Proof. Since outermost MOTS are stable, their curvature is bounded in view of Theorem 2.10. This implies a lower bound on the area of each component. From Theorem 6.5 we thus infer a bound on the number of components.

\section{The Trapped Region}

In this section we examine the weakly outer trapped region, or more precisely the boundary of the weakly outer trapped region. We make the usual assumptions on $(M, g, K)$, that is $(M, g, K)$ is a smooth initial data set with $\partial M$ the disjoint union $\partial M=\partial^{-} M \cup \partial^{+} M$, where $\partial^{-} M$ may be empty, but $\partial^{+} M$ is non-empty, such that $\partial^{ \pm} M$ are smooth, compact surfaces without boundary and $\theta^{+}\left[\partial^{-} M\right]<0$ with respect to the normal pointing into $M$ and $\theta^{+}\left[\partial^{+} M\right]>0$ with respect to the normal pointing out of $M$.

The definition of a trapped set and the trapped region below make sense only if $\theta^{+}\left[\partial^{-} M\right]<0$. However, we can circumvent this requirement for the main theorem as discussed in Remark 7.4 below.

To define the weakly outer trapped region, we introduce the notion of a weakly outer trapped set. 
Definition 7.1. An open set $\Omega \subset M$ with smooth embedded boundary $\partial \Omega$ is called a weakly outer trapped set if $\partial \Omega$ is the disjoint union $\partial \Omega=\partial^{-} M \cup \partial^{+} \Omega$, where $\partial^{+} \Omega$ is a smooth, compact surface without boundary and $\theta^{+}\left[\partial^{+} \Omega\right] \leq 0$ with respect to the normal pointing out of $\Omega$.

Note that $\partial^{+} \Omega$ is homologous to $\partial^{+} M$ in this definition.

Definition 7.2. The weakly outer trapped region is the union of all weakly outer trapped sets enclosing $\partial^{-} M$ :

$$
T:=\bigcup_{\Omega \text { is outer trapped }} \Omega .
$$

We will henceforth refer to $T$ simply as the trapped region. If $\partial^{-} M$ is non-empty, then the trapped region is non-empty as well, but if $\partial^{-} M$ is empty it might happen that $T$ is empty. In this case the statements below are void.

Let $\partial^{-} T:=\partial T \cap \partial^{-} M$ and $\partial^{+} T=\partial T \backslash \partial^{-} M$. The definition of $T$ is analogous

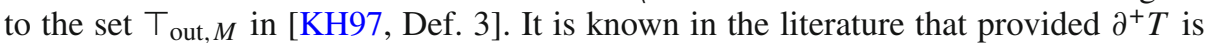
smooth, it satisfies $\theta^{+}=0$ [HE73, KH97]. The most general result about $\partial^{+} T$ we are aware of is [KH97, Prop. 7], which asserts that if $\partial^{+} T$ is $C^{0}$ and piecewise smooth, then it is smooth and satisfies $\theta^{+}=0$. In contrast, we do not assume any initial regularity for $\partial^{+} T$ for the following theorem.

Theorem 7.3. Let $(M, g, K)$ be such that $\partial M$ is the disjoint union $\partial M=\partial^{+} M U$ $\partial^{-} M$ such that $\theta^{+}\left[\partial^{-} M\right]<0$ if $\partial^{-} M$ is non-empty, and $\partial^{+} M$ is non-empty and has $\theta^{+}\left[\partial^{+} M\right]>0$. Let $T$ be the trapped region in $M$. If $T$ is non-empty, then $\partial T$ is the disjoint union $\partial T=\partial^{-} T \cup \partial^{+} T$ of smooth, compact surfaces without boundary, where $\partial^{-} T=\partial^{-} M$ and $\partial^{+} T$ is a smooth stable MOTS homologous to $\partial^{+} M$.

Remark 7.4. If $\left(M^{\prime}, g^{\prime}, K^{\prime}\right)$ is a data set where $\partial^{-} M^{\prime}$ is only a weak barrier $\theta^{+}\left[\partial^{-} M^{\prime}\right] \leq$ 0 , then $\left(M^{\prime}, g^{\prime}, K^{\prime}\right)$ can be modified to $(\tilde{M}, \tilde{g}, \tilde{K})$ such that $\partial^{-} \tilde{M}$ is a strong barrier $\theta^{+}\left[\partial^{-} \tilde{M}\right]<0$. This construction was already used in Sect. 5. The trapped region $\tilde{T} \subset \tilde{M}$ of this extension is such that $\partial^{+} \tilde{T} \subset M^{\prime}$, that is, it lies in $M^{\prime}$, since the region bounded by $\partial^{-} \tilde{M}$ and $\partial^{-} M^{\prime}$ is a trapped set. However, it might be possible that $\partial^{+} \tilde{T} \cap \partial^{-} M^{\prime} \neq \varnothing$. In this case the intersection $\partial^{+} \tilde{T} \cap \partial^{-} M^{\prime}$ is a sub-collection of the components of $\partial^{-} M^{\prime}$ which are stable MOTS.

Remark 7.5. If the dominant energy condition holds, then $\partial^{+} T$ is a collection of spheres or tori [HE73, AK03, GS06].

The proof is along the lines of [HI01, Sect. 4]. Before we begin the proof of the theorem we prove some lemmas, which essentially replace the maximum principle, which is not as powerful for MOTS, as it is for minimal surfaces.

Lemma 7.6. Let $(M, g, K)$ be an initial data set as in Theorem 7.3. Let $\Omega_{1} \subset M$ and $\Omega_{2} \subset M$ be open sets such that $\partial \Omega_{i}$ is the disjoint union $\partial \Omega_{i}=\partial^{-} M \cup \partial^{+} \Omega_{i}$, where $\partial^{+} \Omega_{i}$ is smooth, embedded, and $\partial^{+} \Omega_{i}=\bigcup_{j=1}^{N_{i}} \Sigma_{i}^{j}$ is the union of disjoint, stable, connected MOTS $\Sigma_{i}^{j}, i=1,2$. Then for any $\delta>0$, there exists $\Omega_{1}^{\prime} \subset \Omega_{1}$ and data $K^{\prime}$ on $M$ with the following properties:

1. $\partial \Omega_{1}^{\prime}=\partial^{-} M \cup \partial^{+} \Omega_{1}^{\prime}$,

2. $\partial^{+} \Omega_{1}^{\prime}$ and $\partial^{+} \Omega_{2}$ intersect transversally, 
3. $\operatorname{dist}\left(\partial^{+} \Omega_{1}^{\prime}, \partial^{+} \Omega_{1}\right)<\delta$,

4. $K^{\prime} \in C^{1,1}(M)$ and $K^{\prime}=K$ on $M \backslash \Omega_{1}$,

5. $\theta^{+}$on $\partial^{+} \Omega_{2} \cap M \backslash \Omega_{1}^{\prime}$ computed with respect to $K^{\prime}$ is at most its value with respect to $K$, and

6. there exists a foliation $\Sigma_{s}, s \in(-\varepsilon, 0]$ of $\Omega_{1} \backslash \Omega_{1}^{\prime}$ such that $\Sigma_{0}=\partial^{+} \Omega_{1}$ and $\theta^{+}\left[\Sigma_{s}\right]<$ 0 with respect to the data $K^{\prime}$.

Proof. By pushing the components of $\partial^{+} \Omega_{1}$ into $\Omega_{1}$, as in the proof of Theorem 5.1, while changing the data $K$ to $K^{\prime}$ near components of $\partial \Omega_{1}$ which are stable but not strictly stable, we can construct $K^{\prime}$ and a foliation $\Sigma_{s}$ near $\partial \Omega_{1}$ such that each $\Sigma_{s}$ has $\theta^{+}\left[\Sigma_{s}\right]<0$, thus satisfying Properties 1, 4 and 6. By Sard's theorem, $\Sigma_{s}$ and $\partial^{+} \Omega_{2}$ intersect transversally for almost every $s \in(-\varepsilon, 0)$. Hence we can pick one such $s$, for which also Properties 2 and 3 are satisfied. Property 5 follows by construction, as we were subtracting a non-negative definite tensor from $K$ to obtain $K^{\prime}$.

Subsequently, for two sets $\Omega_{1}, \Omega_{2}$ we denote by $\Omega_{1} \Delta \Omega_{2}$ the symmetric difference, defined by $\Omega_{1} \triangle \Omega_{2}=\left(\Omega_{1} \backslash \Omega_{2}\right) \cup\left(\Omega_{2} \backslash \Omega_{1}\right)$.

Lemma 7.7. Let $(M, g, K), \Omega_{1}$ and $\Omega_{2}$ be as in the previous lemma. Assume furthermore that $\Omega_{1} \triangle \Omega_{2} \neq \emptyset$. Then there exists $\Omega \supset \Omega_{1} \cup \Omega_{2}$, such that $\partial \Omega$ is the disjoint union $\partial \Omega=\partial^{-} M \cup \partial^{+} \Omega$, where $\partial^{+} \Omega$ is an embedded stable MOTS. Any connected component of $\partial^{+} \Omega_{1}$ which intersects $\Omega_{2}$, lies in the interior of $\Omega$.

Proof. There is nothing to prove if $\partial\left(\Omega_{1} \cup \Omega_{2}\right)$ is a smooth embedded manifold. Thus we can assume that $\partial^{+} \Omega_{1}$ and $\partial^{+} \Omega_{2}$ intersect. Fix $\delta>0$ to be the distance at which we can apply Proposition 6.1 in $(M, g, K)$. We use Lemma 7.6, to deform $\Omega_{1}$ and $K$ to $\Omega_{1}^{\prime}$ and $K^{\prime}$ with the stated properties for this choice of $\delta$. As $\partial^{+} \Omega_{1}^{\prime}$ and $\partial^{+} \Omega_{2}$ intersect transversally, Lemma 2.14 allows us to smooth out the corner of $\partial\left(\Omega_{1}^{\prime} \cup \Omega_{2}\right)$ in the outward direction.

Furthermore, all stable components of $\partial^{+} \Omega_{1}$ which were touching $\partial \Omega_{2}$ but not intersecting $\Omega_{2}$ give rise to components of $\partial^{+} \Omega_{1}^{\prime}$, which are disjoint of $\partial^{+} \Omega_{2}$ and at a distance at most $\delta$ to $\partial^{+} \Omega_{2}$. Thus we can apply the surgery procedure of Proposition 6.1 to join these components to $\partial \Omega_{2}$. This yields an open set $\Omega^{\prime}$ with $\Omega^{\prime} \supset \Omega_{1}^{\prime} \cup \Omega_{2}$ and $\partial \Omega^{\prime}$ is the disjoint union $\partial \Omega^{\prime}=\partial^{-} \Omega^{\prime} \cup \partial^{+} \Omega^{\prime}$, where $\partial^{-} \Omega^{\prime}=\partial^{-} M$ and $\partial^{+} \Omega^{\prime}$ is $C^{1,1}$ and has $\theta^{+}\left[\partial^{+} \Omega^{\prime}\right] \leq 0$ and $\theta^{+}\left[\partial^{+} \Omega^{\prime}\right] \not \equiv 0$, as $\theta^{+} \not \equiv 0$ on the components of $\partial^{+} \Omega^{\prime}$ which were created from joining a component of $\partial^{+} \Omega_{1}^{\prime}$ to a component of $\partial^{+} \Omega_{2}$. We can then use the flow from Lemma 5.2 to smooth out the boundary of $\Omega^{\prime}$, yielding $\Omega^{\prime \prime} \supset \Omega^{\prime} \supset \Omega_{1}^{\prime} \cup \Omega_{2}$ with $\Omega^{\prime \prime}$ an open set. Note, by construction all components of $\partial^{+} \Omega_{1}^{\prime}$ and all components of $\partial^{+} \Omega_{2}$ which were joined with components from $\partial^{+} \Omega_{1}^{\prime}$ are contained in the interior of $\Omega^{\prime \prime}$.

Now an application of Theorem 5.1 to the data $\left(M \backslash \Omega^{\prime \prime}, g, K\right)$, with inner boundary $\partial^{-}\left(M \backslash \Omega^{\prime \prime}\right)=\partial^{+} \Omega^{\prime \prime}$, and outer boundary $\partial^{+} M$ yields a set $\Omega \supset \Omega^{\prime \prime}$ with boundary $\partial \Omega$ the disjoint union $\partial \Omega=\partial^{-} M \cup \partial^{+} \Omega$, where $\partial^{+} \Omega$ is a smooth, stable MOTS.

By construction all components of $\partial^{+} \Omega_{1}^{\prime}$ and $\partial^{+} \Omega_{2}$ are in the interior of $\Omega$. Furthermore, an application of the strong maximum principle as in the proof of Theorem 5.1 implies that $\partial^{+} \Omega$ can not penetrate the region $\Omega_{1} \backslash \Omega_{1}^{\prime}$ as this is foliated by trapped surfaces. In particular all components of $\partial^{+} \Omega_{1}$ which meet $\partial^{+} \Omega_{2}$ are contained in the interior of $\Omega$.

Remark 7.8. The preceding lemma implies the uniqueness of outermost MOTS.

Proof of Theorem 7.3. Subsequently we assume that $T$ is non-empty, and therefore $(M, g, K)$ contains trapped regions, as otherwise there is nothing to prove. We first 
show that we can define $\partial^{+} T$ by a collection of sets with much more well-behaved boundaries. We define $\mathcal{T}$ to be the collection of all outer trapped sets $\Omega$, such that the outer boundary $\partial^{+} \Omega$ satisfies the following four assumptions:

1. $\theta^{+}\left[\partial^{+} \Omega\right]=0$;

2. every component of $\partial^{+} \Omega$ is stable, and thus satisfies sup $|A| \leq C$, where $C$ is the constant from Theorem 2.10, and depends only on $\left\|{ }^{M} \mathrm{Rm}\right\|_{C^{0}(M)},\|K\|_{C^{1}(M)}$ and $\operatorname{inj}_{\rho}(M, g, K ; \partial M)$;

3. $i^{+}\left(\partial^{+} \Omega\right) \geq \delta$ where $\delta$ depending on the same data as $C$ above is the $\delta$ from Theorem 6.3;

4. $\left|\partial^{+} \Omega\right| \leq C$, where $C$ is the area resulting from Proposition 6.4 applied to $\partial^{+} \Omega$ with $i^{+}\left(\partial^{+} \Omega\right) \geq \delta$ for the above $\delta$. This $C$ also depends only on $\operatorname{inj}_{\rho}(M, g, K ; \partial M)$, $\left\|{ }^{M} \mathrm{Rm}\right\|_{C^{0}(M)}$ and $\|K\|_{C^{1}(M)}$.

To this end, assume that $\Omega$ is an outer trapped set, which does not lie in $\mathcal{T}$. Then we construct a set $\Omega^{\prime} \supset \Omega$ which lies in $\mathcal{T}$ by applying Theorem 6.3 and using Proposition 6.4 to prove the area estimate.

We thus see that

$$
T=\bigcup_{\Omega \in \mathcal{T}} \Omega .
$$

The first claim is that for each point $p \in \partial^{+} T$ there exists $\Omega \in \mathcal{T}$ such that $p \in \partial^{+} \Omega$. Clearly, for every $n$ there exists $\Omega_{n}$ such that $\operatorname{dist}\left(\Sigma_{n}, p\right)<\frac{1}{n}$, where $\Sigma_{n}=\partial^{+} \Omega_{n}$. We can now appeal to the compactness theorem [AM05, Theorem 1.3] for stable MOTS with bounded curvature and bounded area, which, after passing to a sub-sequence, yields a limit $\Sigma$ of $\partial^{+} \Omega_{n}$ in $C^{1, \alpha}$. This $\Sigma$ is a smooth stable MOTS with bounded curvature and bounded area. Furthermore, $\Sigma$ is the outer boundary of a set $\Omega$, as the $\partial^{+} \Omega_{n}$ can eventually be represented as graphs over $\Sigma$.

However, $\Sigma$ is not necessarily embedded, as the limit of embedded surfaces might meet itself. As $i^{+}\left(\partial^{+} \Omega\right) \geq \delta$, the only thing that prevents $\Sigma$ from being embedded are points where $\Sigma$ touches itself from the inside. To remedy this, we can replace the sequence of the $\Omega_{n}$ by a sequence $\Omega_{n}^{\prime}$ which is increasing in the sense that $\Omega_{n}^{\prime} \subset \Omega_{n+1}^{\prime}$ for all $n$. We proceed inductively and let $\Omega_{1}^{\prime}:=\Omega_{1}$. Assume that we have constructed

$$
\Omega_{1}^{\prime} \subset \Omega_{2}^{\prime} \subset \cdots \subset \Omega_{n-1}^{\prime}
$$

with $\Omega_{k}^{\prime} \in C T$ for $k=1, \ldots, n-1$. Consider the set $\Omega_{n} \cup \Omega_{n-1}^{\prime}$. Either this set has a smooth embedded boundary, in which case we can use Theorem 5.1 to ensure the existence of $\Omega_{n}^{\prime} \supset \Omega_{n} \cup \Omega_{n-1}^{\prime}$ or $\Omega_{n} \cup \Omega_{n-1}^{\prime}$ does not have a smooth boundary. Then Lemma 7.7 yields a barrier for Theorem 5.1 and allows us to construct $\Omega_{n}^{\prime} \supset \Omega_{n} \cup \Omega_{n-1}^{\prime}$. By eventually applying Theorem 6.3, we can assume that $\Omega_{n}^{\prime} \in \mathcal{T}$.

We will now relabel $\Omega_{n}:=\Omega_{n}^{\prime}$ and $\Sigma_{n}:=\Sigma_{n}^{\prime}$. As explained above, there is a subsequence of the $\Omega_{n}$ such that the $\Sigma_{n}$ converge in $C^{1, \alpha}$ to a stable MOTS $\Sigma$ which is the outer boundary of a set $\Omega$ and has $i^{+}(\Sigma) \geq \delta$, thus $\Sigma$ can not touch itself on the outside. Since the $\Omega_{n}$ are increasing, $\Sigma$ can not touch itself on the inside either. This follows from the fact that the $\Sigma_{n}$ converge as graphs from the inside to $\Sigma$. Thus if $\Sigma$ touches itself on the inside, so would the $\Sigma_{n}$. But each $\Sigma_{n}$ is embedded, and hence $\Sigma$ is embedded and $\Omega \in \mathcal{T}$.

Next we show that $\partial^{+} T$ consists of a smooth collection of MOTS. To this end assume first that $\Omega_{1}$ and $\Omega_{2}$ are such that the outer boundaries $\partial^{+} \Omega_{k}$ meet $\partial^{+} T$ for $k=1,2$. 
Let $\Sigma_{k}$ be a component of $\partial^{+} \Omega_{k}$ that meets $\partial T$. From Lemma 7.7 we infer that either $\Sigma_{1}=\Sigma_{2}$ or $\operatorname{dist}\left(\Sigma_{1}, \Sigma_{2}\right)>0$.

It follows that $\partial T$ is a collection of disjoint stable MOTS.

Acknowledgements. The authors wish to thank Walter Simon, Marc Mars, Greg Galloway, Rick Schoen and Gerhard Huisken for helpful conversations. The second author would also like to thank Michael Eichmair and Leon Simon for their comments.

Open Access This article is distributed under the terms of the Creative Commons Attribution Noncommercial License which permits any noncommercial use, distribution, and reproduction in any medium, provided the original author(s) and source are credited.

\section{References}

[AG05] Ashtekar, A., Galloway, G.J.: Some uniqueness results for dynamical horizons. Adv. Theor. Math. Phys. 9(1), 1-30 (2005)

[AK03] Ashtekar, A., Krishnan, B.: Dynamical horizons and their properties. Phys. Rev. D 68(10), 104030 (2003)

[AM05] Andersson, L., Metzger, J.L.: Curvature estimates for stable marginally trapped surfaces. http:// arXiv:org/abs/gr-qc/0512106, 2005

[AMS05] Andersson, L., Mars, M., Simon, W.: Local existence of dynamical and trapping horizons. Phys. Rev. Lett. 95, 111102 (2005)

[AMS07] Andersson, L., Mars, M., Simon, W.: Stability of marginally outer trapped surfaces and existence of marginally outer trapped tubes. http://arXiv:org/abs/0704.2889v2[gr-qc], 2007

[BD04] Ben-Dov, I.: Penrose inequality and apparent horizons. Phys. Rev. D 70(12), 124031 (2004)

$\left[\mathrm{CLZ}^{+}\right.$07] Campanelli, M., Lousto, C.O., Zlochower, Y., Krishnan, B., Merritt, D.: Spin flips and precession in black-hole-binary mergers. Phys. Rev. D 75, 064030 (2007)

[CM99] Colding, T.H., Minicozzi, W.P. II.: Examples of embedded minimal tori without area bounds, internat. Math. Res. Notices 99(20), 1097-1100 (1999)

[Dea03] Dean, B.: Compact embedded minimal surfaces of positive genus without area bounds. Geom. Dedicata 102, 45-52 (2003)

[Eic07] Eichmair, M.: The plateau problem for apparent horizons. http://arXiv:org/abs/0711.4139, 2007

[GS06] Galloway, G.J., Schoen, R.: A generalization of hawking's black hole topology theorem to higher dimensions. Commun. Math. Phys. 266(2), 571-576 (2006)

[GT98] Gilbarg, D., Trudinger, N.S.: Elliptic partial differential equations of second order. Rev. 3. printing. Second ed., Berlin-Heidelberg-New York: Springer-Verlag, 1998

[HE73] Hawking, S.W., Ellis, G.F.R.: The large scale structure of space-time. Cambridge Monographs on Mathematical Physics, No. 1. London: Cambridge University Press, 1973

[HI01] Huisken, G., Ilmanen, T.: The inverse mean curvature flow and the riemannian penrose inequality. J. Differ. Geom. 59(3), 353-437 (2001)

[HP99] Huisken, G., Polden, A.: Geometric evolution equations for hypersurfaces. In: Calculus of variations and geometric evolution problems (Cetraro, 1996), Lecture Notes in Math., vol. 1713, Berlin: Springer, 1999, pp. 45-84

[Jan78] Jang, P.S.: On the positivity of energy in general relativity. J. Math. Phys. 19, 1152-1155 (1978)

[KH97] Kriele, M., Hayward, S.A.: Outer trapped surfaces and their apparent horizon. J. Math. Phys. 38(3), 1593-1604 (1997)

[KLZ07] Krishnan, B., Lousto, C.O., Zlochower, Y.: Quasi-local linear momentum in black-hole binaries. Phys. Rev. D 76, 081501 (2007)

[Lie96] Lieberman, G.M.: Second order parabolic differential equations. River Edge, NJ: World Scientific Publishing Co. Inc., 1996

[NR06] Nabutovsky, A., Rotman, R.: Curvature-free upper bounds for the smallest area of a minimal surface. Geom. Funct. Anal. 16(2), 453-475 (2006)

[Sch04] Schoen, R.: Talk given at the Miami Waves conference. January 2004

[SSY75] Schoen, R., Simon, L., Yau, S.T.: Curvature estimates for minimal hypersurfaces. Acta Math. 134(3-4), 275-288 (1975)

[SY81] Schoen, R., Yau, S.-T.: Proof of the positive mass theorem. II. Commun. Math. Phys. 79(2), 231-260 (1981)

[SY94] Schoen, R., Yau, S.-T.: Lectures on differential geometry. Conference Proceedings and Lecture Notes in Geometry and Topology, Boston: International Press, 1994

[Yau01] Yau, S.-T.: Geometry of three manifolds and existence of black hole due to boundary effect. Adv. Theor. Math. Phys. 5(4), 755-767 (2001) 